\title{
Resveratrol regulates mitochondrial reactive oxygen species homeostasis through Sirt3 signaling pathway in human vascular endothelial cells
}

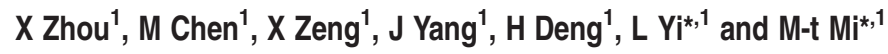

Mitochondrial reactive oxygen species (mtROS) homeostasis plays an essential role in preventing oxidative injury in endothelial cells, an initial step in atherogenesis. Resveratrol (RSV) possesses a variety of cardioprotective activities, however, little is known regarding the effects of RSV on mtROS homeostasis in endothelial cells. Sirt3 is a mitochondrial deacetylase, which plays a key role in mitochondrial bioenergetics and is closely associated with oxidative stress. The goal of the study is to investigate whether RSV could attenuate oxidative injury in endothelial cells via mtROS homeostasis regulation through Sirt3 signaling pathway. We found that pretreatment with RSV suppressed tert-butyl hydroperoxide (t-BHP)-induced oxidative damage in human umbilical vein endothelial cells (HUVECs) by increasing cell viability, inhibiting cell apoptosis, repressing collapse of mitochondrial membrane potential and decreasing mtROS generation. Moreover, the enzymatic activities of isocitrate dehydrogenase 2 (IDH2), glutathione peroxidase (GSH-Px) and manganese superoxide dismutase (SOD2) as well as deacetylation of SOD2 were increased by RSV pretreatment, suggesting RSV notably enhanced mtROS scavenging in t-BHP-induced endothelial cells. Meanwhile, RSV remarkably reduced mtROS generation by promoting Sirt3 enrichment within the mitochondria and subsequent upregulation of forkhead box 03A (Fox03A)-mediated mitochondria-encoded gene expression of ATP6, C01, Cytb, ND2 and ND5, thereby leading to increased complex I activity and ATP synthesis. Furthermore, RSV activated the expressions of phosphorylated adenosine monophosphate-activated protein kinase (p-AMPK), peroxisome proliferator-activated receptor gamma coactivator-1 $\alpha$ (PGC-1 $\alpha$ ) and Sirt3, as well as estrogen-related receptor- $\alpha$ (ERR $\alpha$ )-dependent Sirt3 mRNA transcription, which were abolished in the presence of AMPK inhibitor and AMPK, PGC-1 $\alpha$ or Sirt3 siRNA transfection, indicating the effects of RSV on mtROS homeostasis regulation were dependent on AMPK-PGC-1 $\alpha$-ERR $\alpha$-Sirt3 signaling pathway. Our findings indicated a novel mechanism that RSVattenuated oxidative injury in endothelial cells through the regulation of mtROS homeostasis, which, in part, was mediated through the activation of the Sirt3 signaling pathway.

Cell Death and Disease (2014) 5, e1576; doi:10.1038/cddis.2014.530; published online 18 December 2014

Atherosclerotic cardiovascular disease remains the main
cause of death in the United States. Numerous studies have
demonstrated that atherosclerosis (AS) is the result of a
prolonged and excessive response to injury in the vascular
wall, which begins with oxidative damage in the endothelium,
characterized by endothelial dysfunction. ${ }^{1}$ Oxidative stress,
which can be defined as an imbalance between the production
of endogenous reactive oxygen species (ROS) and the presence of antioxidant molecules, is one of the most important mechanisms contributing to endothelial dysfunction. ${ }^{2}$ It has been recognized that the mitochondria are the main source of ROS intracellularly and cardiovascular risk factors are associated with excessive mitochondrial ROS (mROS) production that promotes oxidative stress and inflammation, and decreases nitric oxide bioavailability. Moreover, vascular endothelial cells are highly glycolytic and

\footnotetext{
${ }^{1}$ Research Center for Nutrition and Food Safety, Chongqing Key Laboratory of Nutrition and Food Safety, Institute of Military Preventive Medicine, Third Military Medical University, Chongqing Medical Nutrition Research Center, Chongqing 400038, PR China

${ }^{*}$ Corresponding author: LYi or M-t Mi, Research Center for Nutrition and Food Safety, Chongqing Key Laboratory of Nutrition and Food Safety, Institute of Military Preventive Medicine, Third Military Medical University, Chongqing Medical Nutrition Research Center, 30th Gaotanyan Main Street, Shapingba District, Chongqing 400038, PR China. Tel/Fax: +86 2368752307; E-mail: longgyin8341@ hotmail.com (LY) or Tel/Fax: +86 2368752305; E-mail: mi_mt2009@ hotmail.com (M-tM)

Abbreviations: ACO, aconitase; AIF, apoptosis-inducing factor; AMPK, adenosine monophosphate-activated protein kinase; AS, atherosclerosis; ATP, adenosine triphosphate; ATP6, ATP synthase 6; Bax, Bcl-2-associated X protein; Bcl-2, B-cell lymphoma-2; BSA, bovine serum albumin; CAD, cardiovascular disease; CCK-8, cell counting kit; cDNA, complementary DNA; ChIP, Chromatin immunoprecipitation; CO1, cytochrome $c$ oxidase pseudogene 1; Cytb, cytochrome $b$; Cytc, Cytochrome $c$; DAPI, 4', 6'-diamidino-2-phenylindole; DMSO, dimethylsulfoxide; ETC, electron transport chain; ETFDH, electron transfer flavoprotein dehydrogenase; ERR $\alpha$, estrogenrelated receptor $\alpha$; ERRE, estrogen receptor response element; FITC, fluorescein isothiocyanate; FoxO3A, Forkhead box O3A; GAPDH, glyceraldehyde-3-phosphate dehydrogenase; GPD2, glycerol phosphate dehydrogenase; GSSG, oxidized glutathione; GSH-Px, glutathione peroxidase; HUVECs, human umbilical vein endothelial cells; IDH2, isocitrate dehydrogenase 2; JC-1, 5,5,'6,6'-tetrachloro-1,1',3,3'-tetraethylbenzimidazolylcarbocyanine iodide; KGDH, $\alpha$-keto glutaric dehydrogenase; mtROS, mitochondrial reactive oxygen species; $\mathrm{NAD}^{+}$, nicotinamide adenine nucleotide, oxidized form; NADPH, nicotinamide adenine dinucleotide, reduced form; ND2, NADH dehydrogenase 2; ND5, NADH dehydrogenase 5; OXPHOS, oxidative phosphorylation; PDH, pyruvate dehydrogenase; PGC-1 $\alpha$, peroxisome proliferator-activated receptor- $\gamma$ coactivator-1 $\alpha$; PI, propidium iodide; ROS, reactive oxygen species; RSV, resveratrol; SDS-PAGE, sodium dodecyl sulfatepolyacrylamide gel; siRNA, small-interfering RNA; Sirt3, silent mating-type information regulation 2 homolog 3; SOD2, manganese superoxide dismutase; t-BHP, tert-butyl hydroperoxide; TCA, tricarboxylic acid; $\Delta y m$, mitochondrial membrane potential

Received 28.4.14; revised 06.10.14; accepted 04.11.14; Edited by M Campanella
} 
the mitochondrial content is relatively low in comparison with other cell types with higher energy requirements. ${ }^{3-5}$ Thus, it was indicated that the mitochondria in endothelial cells are to play a limited role in energy production but, sense the local environment the endothelial cells face and orchestrate the cellular homeostasis and function by virtue of its ROSproducing capacity. ${ }^{6}$ In the normal condition, the mtROS participate in critical signaling pathways to mediate adaptive responses and regulate diverse biological functions. However, excess mtROS production can directly induce endothelial dysfunction through stimulating the production of proinflammatory cytokines. Thus, development of drugs, which maintain mtROS homeostasis could be crucial for preventing endothelial dysfunction.

Resveratrol (3, 4', 5-trihydroxystilbene, RSV) is a polyphenolic compound found mainly in the skins of grapes, and berry fruits (cranberry, strawberry, mulberry, etc.), and in peanuts and red wine. Accumulating evidence suggests that $\mathrm{RSV}$ is a highly pleiotropic molecule that modulates abundant targets and thus influences numerous biochemical and molecular functions. ${ }^{7}$ Epidemiological studies indicate that the Mediterranean diet, which is rich in RSV, is associated with a reduced risk of carotid $A S^{8}{ }^{8}$ Evidence from clinical trials indicated that RSV may protect against AS by improving gene expression in the vascular endothelium, and could be considered as a preventive agent. ${ }^{9}$ We previously found that RSV could penetrate into vascular endothelial cells and attenuate endothelial inflammation by inducing autophagy through the cyclic adenosine monophosphate (cAMP) signaling pathway. ${ }^{10,11}$ Nonetheless, little information is available regarding the effects of RSV on mtROS homeostasis regulation in endothelial cells.

Recent studies suggested that silent mating-type information regulation 2 homolog 3 (Sirt3), plays a key role in regulating mtROS homeostasis. ${ }^{12}$ Sirt3 is targeted to the mitochondrial matrix where it orchestrates mitochondrial oxidative metabolism, such as the TCA cycle and oxidative phosphorylation (OXPHOS), through deacetylation of a variety of substrates. Intriguingly, Sirt3 also governs the level of mtROS by deacetylation of the major mitochondrial antioxidant enzymes, including manganese superoxide dismutase (SOD2), isocitrate dehydrogenase 2 (IDH2) and glutathione peroxidase (GSH-Px), as well as the components for mtROS generation in the electron transport chain (ETC) such as complex I and complex III. ${ }^{13,14}$ Moreover, it has been reported that Sirt3 functions as a downstream target of peroxisome proliferator-activated receptor (PPAR $\gamma$ ) coactivator-1a (PGC-1a), which is directly regulated by adenosine monophosphate-activated protein kinase (AMPK), thereby stimulating deacetylation of mitochondrial enzymes involved in mtROS homeostasis and activation of forkhead box O3A (FoxO3A)-mediated mitochondrial DNA (mtDNA) transcription to attenuate oxidative damage. ${ }^{12,15,16}$ Recent work highlights the critical importance of Sirt3 in the regulation of energetic metabolism by RSV treatment in the liver through stimulating complex I activity. ${ }^{17}$ Thus, we hypothesized that RSV could attenuate oxidative injury in endothelial cells via mtROS homeostasis regulation through Sirt3 signaling pathway. As expected, our results indicated, for the first time, that RSV-attenuated oxidized injury in endothelial cells induced by oxidative damage through regulation of mtROS homeostasis, which, in part, was mediated through the activation of the Sirt3 signaling pathway. These results provide new evidence regarding the vascular protective effects of RSV and may also open new avenues for finding new drugs that can be applied to endothelial protection and AS prevention.

\section{Results}

RSV inhibited t-BHP-induced endothelial injury in human umbilical vein endothelial cells. As shown in Figures 1a and b, tert-butyl hydroperoxide (t-BHP) decreased cell viability in a dose-dependent (Figure 1a) and timedependent (Figure 1b) manner in human umbilical vein endothelial cells (HUVECs), respectively. The cell viability was reduced to $52 \pm 2.16 \%$ when treated with t-BHP $(80 \mu \mathrm{M})$ for $4 \mathrm{~h}$ compared to the control group. However, RSV pretreatment significantly attenuated the detrimental effect of t-BHP on cell viability and apoptosis in HUVECs $(P<0.05$; Figures $1 \mathrm{c}-\mathrm{e})$. And RSV $(0.1,1,10$ and $15 \mu \mathrm{M})$ alone had no notable effects on cell viability and apoptosis in HUVECs $(P>0.05 ;$ Figures $1 \mathrm{c}-\mathrm{e})$. Moreover, the expressions of apoptosis-related factors including apoptosis-inducing factor (AIF), cytochrome $c$ (Cytc), Bcl-2-associated $X$ protein (Bax) and B-cell lymphoma-2 (Bcl-2) were measured by flow cytometry. As shown in Figures 1f-h, the expression of Bax was decreased significantly in the mitochondrial fraction but increased significantly in the cytosolic fraction in HUVECs when treated with $\mathrm{t}-\mathrm{BHP}$, compared with the control. However, $\mathrm{Bcl}-2$ decreased notably in the cytosolic fraction as a result of t-BHP treatment, while there seemed no significant changes of $\mathrm{Bcl}-2$ expressions in the mitochondrial fractions. Consequently, the $\mathrm{Bax} / \mathrm{Bcl}-2$ ratio after t-BHP treatment decreased significantly in the mitochondrial fraction, but increased in the cytosolic fraction in HUVECs, while resveratrol pretreatment inhibited $\mathrm{t}-\mathrm{BHP}$-induced changes of $\mathrm{Bax} / \mathrm{Bcl}-2$ ratio both in cytosolic and mitochondrial fractions. Meanwhile, RSV pretreatment decreased the cytosolic expressions of AIF and Cytc in t-BHP-induced endothelial cells (Figures $1 \mathrm{i}$ and $\mathrm{j}$ ). Taken together, these results suggest that RSV pretreatment remarkably attenuated $\mathrm{t}$-BHP-induced oxidative injury in HUVECs.

RSV attenuated t-BHP-induced collapse of mitochondrial membrane potential in HUVECs. The effect of RSV on t-BHP-induced mitochondrial membrane potential $(\Delta \psi \mathrm{m})$ in HUVECs was measured using JC-1 probe. JC-1 can selectively enter mitochondria and reversibly changes color as the $\Delta \psi \mathrm{m}$ changes. ${ }^{18}$ As shown in Figures $2 \mathrm{a}$ and $\mathrm{b}$, there was a significantly increased green fluorescence in cells exposed to t-BHP $(80 \mu \mathrm{M} ; P<0.05)$, suggesting that the mitochondrial membrane was depolarized. However, RSV $(10 \mu \mathrm{M})$ pretreatment repressed the changes of $\Delta \psi \mathrm{m}$ induced by $\mathrm{t}-\mathrm{BHP}$, indicated by a reduction of green fluorescence and restoration of red fluorescence. Moreover, Sirt3 siRNA transfection abolished the effects of RSV on t-BHP-induced changes of $\triangle \psi \mathrm{m}$ in HUVECs. These results suggested that RSV remarkably suppressed t-BHP-induced collapse of 

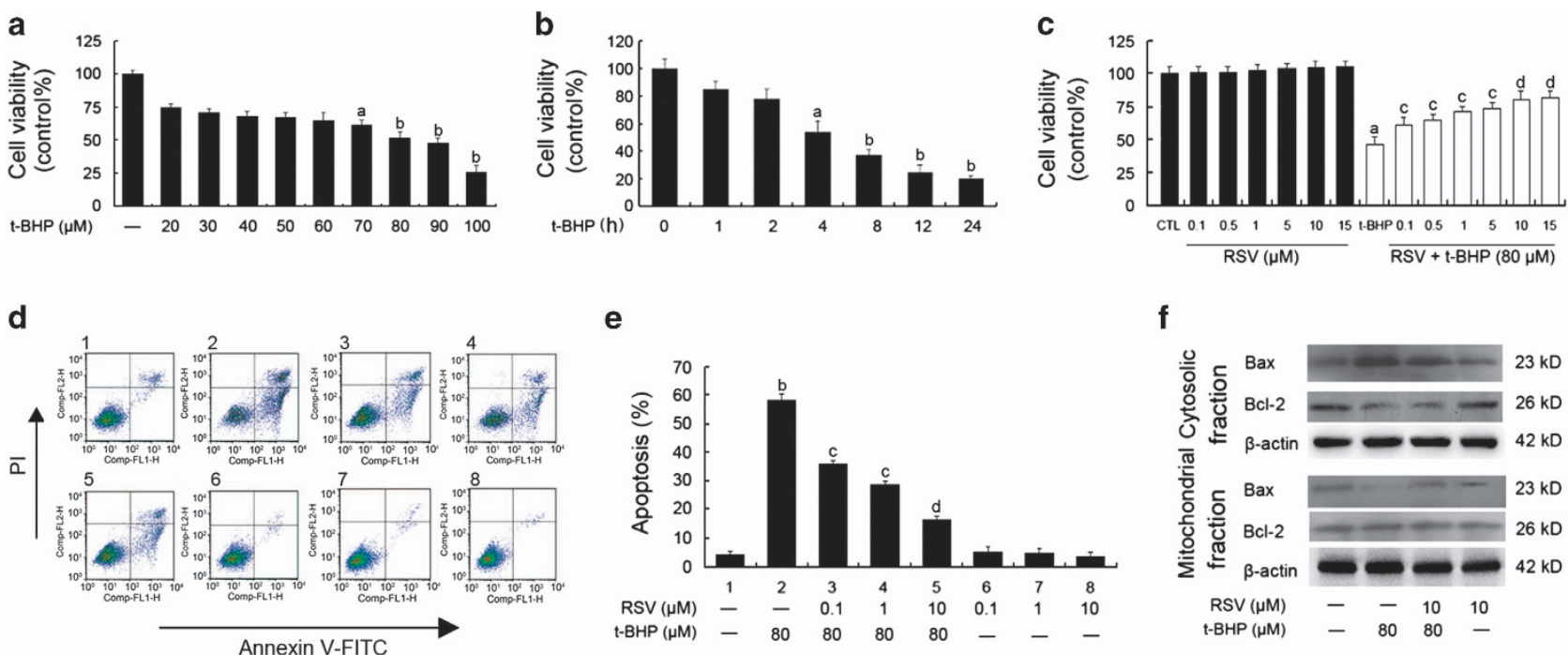

e
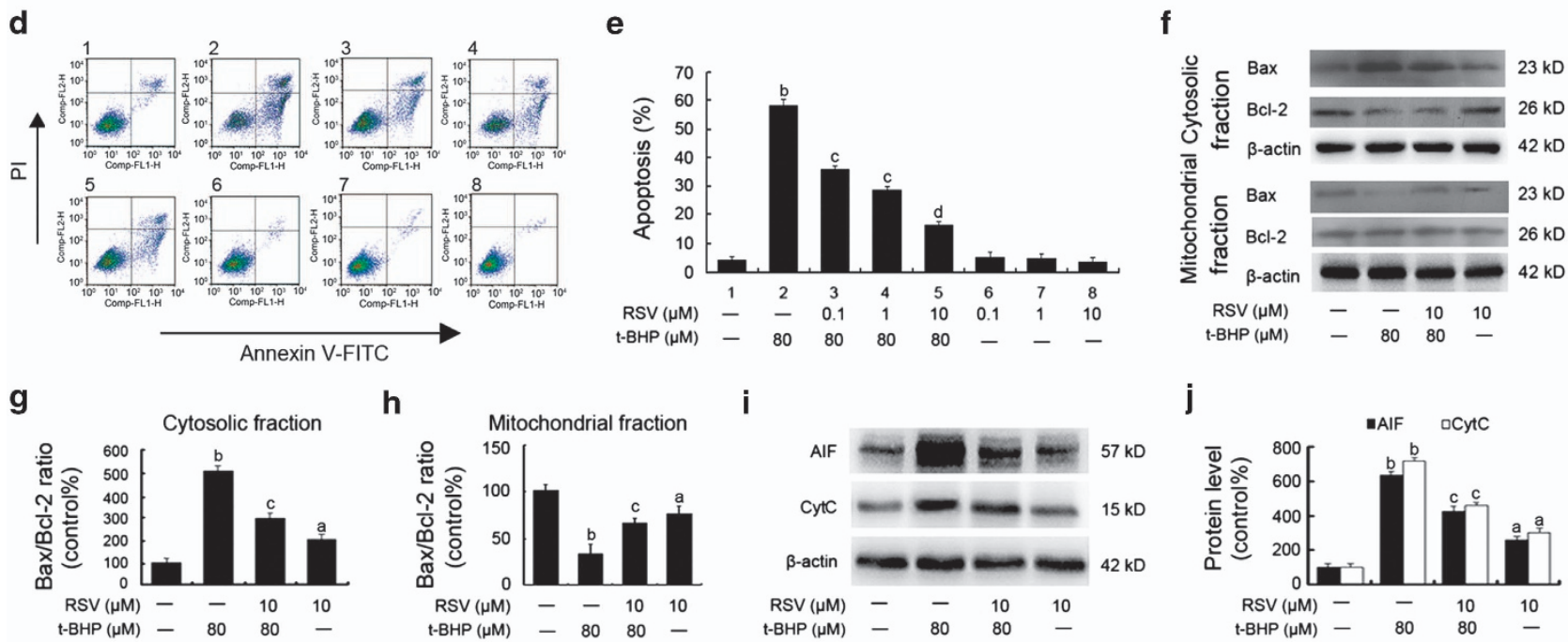

Figure 1 RSV inhibited t-BHP-induced injury in HUVECs. (a and b) Cells were treated with t-BHP with different concentrations $(20,30,40,50,60,70,80,90$ and $100 \mu \mathrm{M})$ for $4 \mathrm{~h}$ (a) or for different time intervals $(1,2,4,8,12$ and $24 \mathrm{~h} ; \mathbf{b})$, respectively. Cell viability was determined using the CCK-8 assay and data are expressed as percentage of the control. (c) Confluent cells were pretreated for $2 \mathrm{~h}$ with various concentrations of RSV $(0.1,0.5,1,5,10$ and $15 \mu \mathrm{M})$. After removing the supernatants, cells were incubated with fresh medium in the presence or absence of t-BHP $(80 \mu \mathrm{M})$ for an additional $4 \mathrm{~h}$. Cell viability was determined using the CCK-8 assay. (d) Representative images of flow cytometric analysis by annexin V-FITC/PI dual staining. The bottom right quadrant represents annexin V-FITC-stained cells (early-phase apoptotic cells) and the top right quadrant represents PI- and annexin V-FITC-dual-stained cells (late-phase apoptotic/necrotic cells). (e) Apoptotic cells are represented as the percentage of annexin-V singlepositive plus annexin-V/PI double-positive cells. (f) After the indicated treatments, the cells were harvested and lysed to detect the cytoplasmic and mitochondrial levels of Bax and Bcl-2 by western blot analysis. (g) The bar graphs show the ratio of Bax/Bcl-2 in the cytosolic fraction in endothelial cells. (h) The bar graphs show the ratio of Bax/Bcl-2 in the mitochondrial fraction in endothelial cells. (i) The expressions of AIF and CytC in the cytosolic fraction of endothelial cells were detected. (j) The bar graphs show the quantification of the indicated proteins. All results are representative of three independent experiments and values are presented as means \pm S.E.M. $(n=3)$. ${ }^{a} P<0.05$, ${ }^{b} P<0.01$ versus the control group; ${ }^{\mathrm{c}} \mathrm{P}<0.05,{ }^{\mathrm{d}} P<0.01$ versus the t-BHP-treated group

mitochondrial membrane potential in a Sirt3-dependent manner in HUVECs.

RSV attenuated t-BHP-induced mitochondrial dysfunction by inhibiting mtROS generation in HUVECs. The effects of RSV on regulation of mitochondrial redox status was evaluated using MitoSOX Red (Invitrogen, Carlsbad, CA, USA), a highly selective fluorescent probe for detection of the $\mathrm{O}_{2}^{:-}$generated within mitochondria. The MitoSOX Red reagent is live-cell permeant, and is selectively targeted to mitochondria. Once in mitochondria, MitoSOX Red is oxidized by $\mathrm{O}_{2}^{\bullet-}$ and exhibits red fluorescence. ${ }^{19}$ As shown in Figures $3 a$ and $b$, t-BHP significantly increased mitochondrial $\mathrm{O}_{2}^{\bullet-}$ generation compared with the control group, which was notably reduced by RSV pretreatment $(P<0.05)$. Moreover, this effect of RSV was sharply abolished by Sirt3 siRNA transfection.

RSV reduced mtROS generation by stimulating Sirt3mediated mitochondrial enzyme activities and SOD2 deacetylation. The enzymatic activities of IDH2, SOD2 and GSH-Px that are involved in mtROS scavenging in endothelial cells were measured in this study. As shown in Figures $4 \mathrm{a}-\mathrm{c}$, t-BHP led to sharply decreased enzymatic activities of IDH2, SOD2 and GSH-Px, while RSV pretreatment significantly recovered these enzymatic activities $(P<0.05)$. In addition, western blot analysis of mitochondrial extracts with an acetyl-SOD2 (ac-SOD2) antibody revealed that $\mathrm{t}-\mathrm{BHP}$ increased the acetylation of SOD2, which was suppressed by RSV pretreatment (Figure 4d). However, the effects of RSV on radicals scavenging in HUVECs were significantly abolished by Sirt3 siRNA transfection, indicating that Sirt3 plays a key role in the scavenging of excessive mtROS in HUVECs treated with RSV.

RSV reduced mtROS generation through upregulation of Sirt3-mediated mtDNA transcription. Previous findings showed that FoxO3A co-precipitated with Sirt3 in the mitochondrial fractions of mammalian cells. ${ }^{20}$ Sirt3 has been shown to efficiently deacetylate FoxO3A, which is essential for the regulation of FoxO3A DNA-binding ability. ${ }^{15}$ In the current study, we found that RSV increased Sirt3 (green 
a

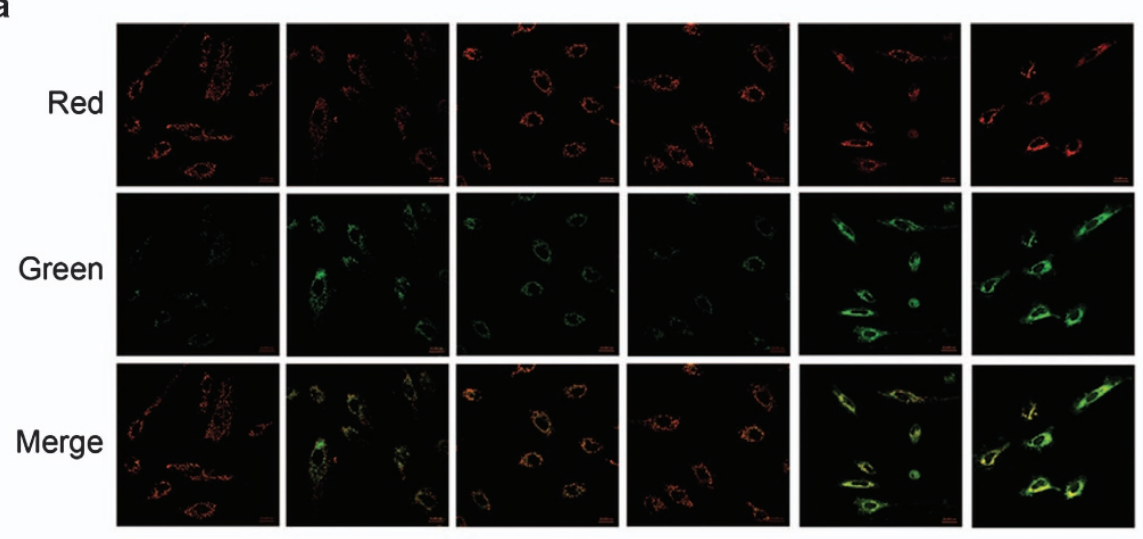

b

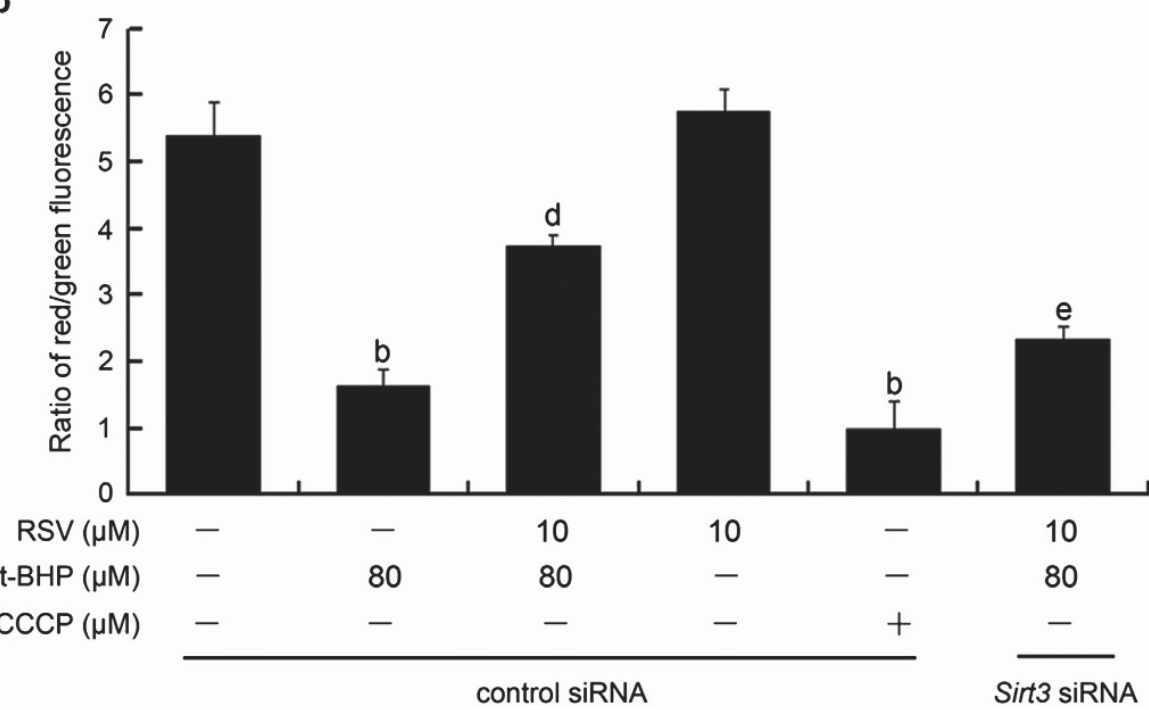

Figure 2 RSV suppressed t-BHP-induced collapse of mitochondrial membrane potential in HUVECs. Sirt3 was knocked down by Sirt3 siRNA transfection as described in the Materials and Methods section. At 24-h post-transfection, cells were pretreated with RSV of $10 \mu \mathrm{M}$ for $2 \mathrm{~h}$, washed, and then treated with or without t-BHP of $80 \mu \mathrm{M}$ for an additional $4 \mathrm{~h}$. (a) Determination of $\Delta y \mathrm{~m}$ was carried out using CLSM. Red fluorescence was emitted by JC-1 aggregates in healthy mitochondria with polarized inner mitochondrial membranes, whereas green fluorescence was emitted by cytosolic JC-1 monomers, indicating $\Delta y \mathrm{~m}$ dissipation. Merged images indicate co-localization of JC-1 aggregates and monomers. (b) $\Delta y \mathrm{~m}$ in each group was calculated as the ratio of red to green fluorescence. All results are presented as mean \pm S.E.M. of at least three independent experiments. ${ }^{b} P<0.01$ versus the control group; ${ }^{d} P<0.01$ versus the t-BHP-treated group; ${ }^{e} P<0.05$ versus RSV $(10 \mu \mathrm{M})$ and t-BHP $(80 \mu \mathrm{M})$ co-treated group with control siRNA transfection

fluorescence by immunofluorescence assay) expression in mitochondria (red fluorescence with Mito-tracker Red) in HUVECs, supporting the hypothesis that RSV stimulates Sirt3 enrichment in mitochondria (Figures $5 a$ and b). RSV addition also induced the expressions of mtRNA polymerase (mtRNAPol) and FoxO3A within mitochondria (Figures $5 \mathrm{c}$ and d). The chromatin immunoprecipitation (ChIP) analysis showed that FoxO3A and Sirt3 were co-recruited to mtDNA by RSV treatment, leading to the upregulation of mtDNA genes including ATP synthase 6 (ATP6), cytochrome $c$ oxidase pseudogene 1 (CO1), cytochrome $b$ (Cytb), NADH dehydrogenase 2 (ND2) and $\mathrm{NADH}$ dehydrogenase 5 (ND5; Figures 5e and f). Moreover, exposure to t-BHP resulted in a significant loss of complex I activity (Figure $5 \mathrm{~g}$ ) and decreased ATP synthesis (Figure 5h) in HUVECs $(P<0.05)$, whereas pretreatment with RSV notably ameliorated t-BHP-induced decrease in mitochondrial ETC function. Furthermore, Sirt3 siRNA transfection decreased the stimulatory effects of RSV on the mtDNA transcription and the coded gene expressions, along with the complex I activity and ATP content, which are possibly attributable to decreased deacetylation of FoxO3A mediated by Sirt3. ${ }^{21}$ These results clearly indicated that RSV pretreatment caused Sirt3 enrichment in mitochondria, which was required for the activation of mtDNA transcription and ATP synthesis, finally leading to a reduction of mtROS as the byproduct of OXPHOS in endothelial cells.

RSV activated AMPK-PGC-1a-ERR $a$-Sirt3 signaling pathway in HUVECs. As shown in Figures $6 a$ and $b$, treatment with RSV alone increased the protein expressions of p-AMPK, PGC-1 $a$ and Sirt3, which were in parallel with 
a
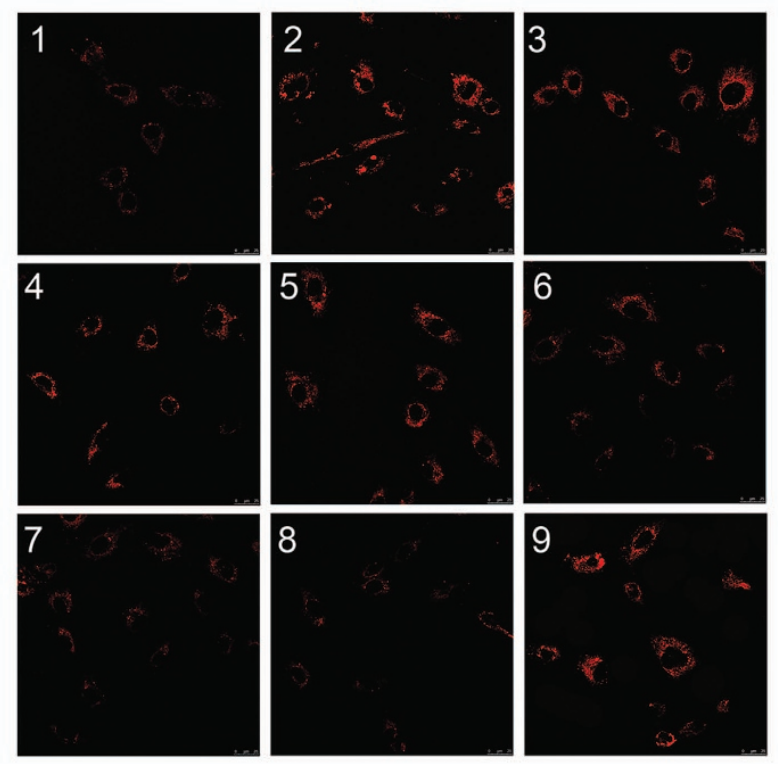

b

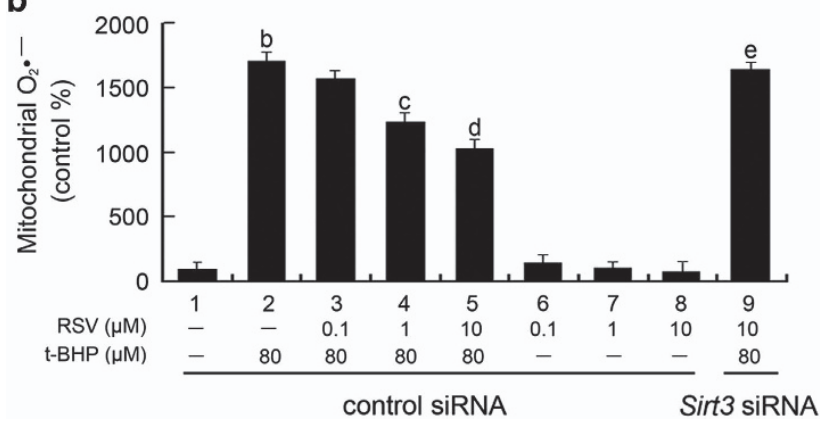

Figure 3 RSV attenuated t-BHP-induced mitochondrial dysfunction by inhibiting mtROS generation in HUVECs. Cells were transfected with Sirt3 siRNA as described in the Materials and Methods section. At 24-h post-transfection, cells were pretreated with RSV $(0.1,1$ and $10 \mu \mathrm{M})$ for $2 \mathrm{~h}$, washed, and then incubated with fresh medium in the presence or absence of t-BHP $(80 \mu \mathrm{M})$ for an additional $4 \mathrm{~h}$. (a) The mitochondrial $\mathrm{O}_{2}^{--}$levels were estimated using MitoSOX Red and the fluorescence values were read at an excitation wavelength of $510 \mathrm{~nm}$ and emission wavelength of $579 \mathrm{~nm}$. (b) The bar charts show quantification of the mitochondrial $\mathrm{O}_{2}^{--}$levels expressed as the fold change relative to the control group. All results are presented as means \pm S.E.M. of at least three independent experiments. ${ }^{b} P<0.01$ versus the control group; ${ }^{\mathrm{C}} P<0.05,{ }^{\mathrm{d}} P<0.01$ versus the t-BHP-treated group; ${ }^{\mathrm{e}} P<0.05$ versus RSV $(10 \mu \mathrm{M})$ and $\mathrm{t}-\mathrm{BHP}(80 \mu \mathrm{M})$ co-treated group with control siRNA transfection

increased Sirt3 mRNA expression (Figure 6c). However, $P G C$-1a siRNA transfection prevented the induction of Sirt3 mRNA and protein expression in HUVECs (Figures 6c and d), indicating that PGC-1a was required for the activation of RSV on Sirt3 expression. And the role of AMPK was further evaluated by the use of an AMPK inhibitor and agonist, as well as AMPK siRNA transfection. RSV-triggered AMPK activation was inhibited in the presence of compound $\mathrm{C}$ (a potent AMPK inhibitor) of $10 \mu \mathrm{M}$ or AMPK SiRNA, accompanied by decreased expression of PGC-1 $a$ and Sirt3. In contrast, chemical activation of AMPK by AICAR $(500 \mu \mathrm{M})$ resulted in increased expression of phosphorylated AMPK (p-AMPK) and PGC-1a (Figures $6 e$ and f). These results implicated that the AMPK-PGC-1a-Sirt3 axis was essential for the effect of RSV on mtROS homeostasis regulation in endothelial cells.

Furthermore, it has been demonstrated that estrogenrelated receptor- $a$ (ERR $a$ ) not only acts as a downstream target of PGC-1a, but is also co-activated by this transcriptional coactivator. ${ }^{22}$ Thus, we performed the luciferase assay to determine if Sirt3 mRNA activation by RSV occurred via PGC-1a-dependent ERR $a$ binding to the Sirt3 promoter. We transfected HUVECs with an ERRE-luc reporter containing fragment of the Sirt3 promoter fused to a luciferase reporter gene. No firefly luciferase activities were measured in the vehicle group, suggesting that luciferase activities in cells cotransfected with ERRE-luc and $\mathrm{pRL}-\mathrm{TK}$ vector were specific. As shown in Figure $6 \mathrm{~g}, \mathrm{t}-\mathrm{BHP}$ exposure resulted in a decreased luciferase activity compared with the control group $(P<0.05)$. In contrast, RSV pretreatment significantly increased ERRE-mediated Sirt3 transcriptional activity, whereas this effect was diminished by addition of PGC-1a siRNA. These results revealed that RSV activated Sirt3 mRNA transcription via a PGC-1a-dependent ERRa-mediated signaling pathway.

In addition, we performed a ChIP assay (Thermo Scientific, Waltham, MA, USA) to confirm weather RSV activated the binding of ERRa to the Sirt3 promoter. As shown in Figure 6h, t-BHP inhibited the amplification of a Sirt3 promoter fragment containing ERRE, while RSV pretreatment increased its amplification, an effect that was abolished by ERRa siRNA transfection. The findings clearly indicated that ERRa played an important role in mediating PGC-1a-induced Sirt3 expression. Overall, the AMPK-PGC-1a-ERRa-Sirt3 signaling pathway appears to be required for RSV-induced mtROS homeostasis and subsequent oxidative injury in endothelial cells.

\section{Discussion}

The results of this study, for the first time, support a model whereby RSV triggers AMPK-PGC-1a signaling, which is required for ERRa-dependent Sirt3 transcription and subsequent enrichment within the mitochondria. This in turn leads to deacetylation and activation of mitochondrial radical scavenging enzymes and mtDNA-encoded gene transcription and ATP synthesis, finally contributing to mtROS homeostasis in HUVECs (Figure 7). Our findings demonstrate a key role for Sirt3 in the inhibitory effects of RSV on the oxidative injury in endothelial cells, thus providing new insights into the cardiovascular benefits of RSV.

Increased oxidative stress plays an important role in the pathogenesis of cardiovascular diseases. The current study focused on the role of RSV in oxidative stress-induced endothelial cells. Oxidative stress is resulted from perturbation of cellular redox homeostasis, which is further associated with numerous deleterious consequences for the cell (e.g., lipid peroxidation or even cell death), and may play a critical role in endothelial dysfunction. ${ }^{14}$ In this study, t-BHP, a short chain analog of lipid hydroperoxides, was used as a pro-oxidant agent to induce oxidative stress in endothelial cells. Peroxy radicals can be generated from $\mathrm{t}-\mathrm{BHP}$ in the cytosol by its interaction with ferrous iron in a reaction similar to the Fenton 
a

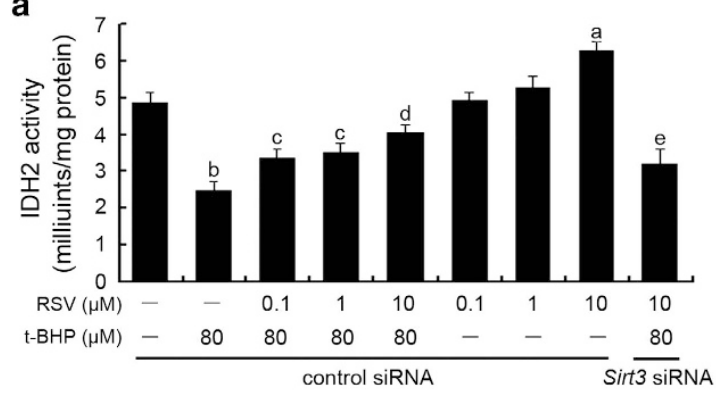

C

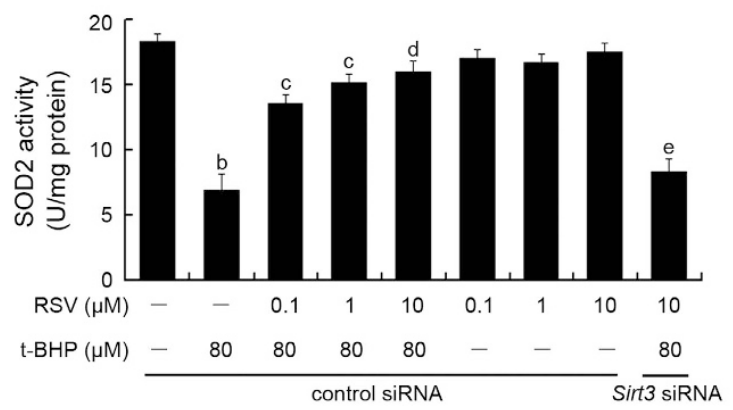

b

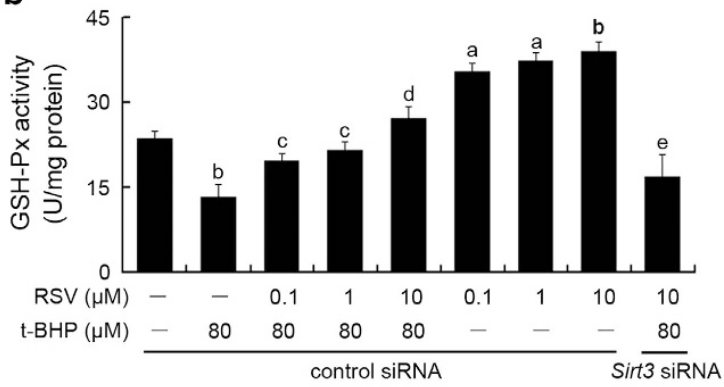

d

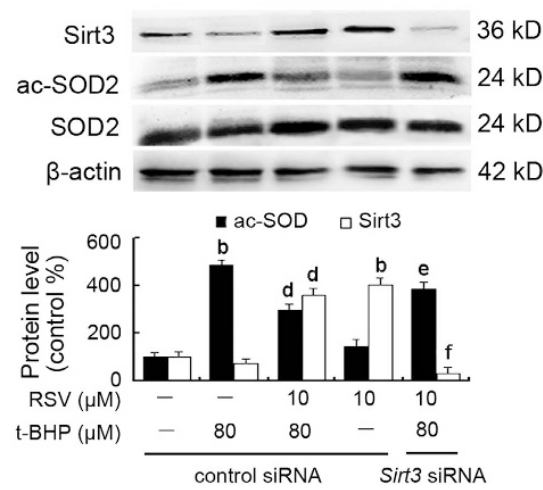

Figure 4 RSV reduced mtROS generation by stimulating Sirt3-mediated mitochondrial enzyme activities and SOD2 deacetylation. Cells were transfected with Sirt3 siRNA as described in the Materials and Methods section. At 24-h post-transfection, cells were pretreated with different concentrations of RSV $(0.1,1$ and $10 \mu \mathrm{M})$ for $2 \mathrm{~h}$ and then treated with or without t-BHP of $80 \mu \mathrm{M}$ for an additional $4 \mathrm{~h}$. (a-c) The enzyme activities of IDH2 (a), GSH-Px (b) and SOD2 (c) were determined using the corresponding assay kits, according to the manufacturer's instructions. (d) After the indicated treatments, the cells were harvested and lysed to detect protein levels of ac-SOD2 by western blot analysis. All results are presented as means \pm S.E.M. of at least three independent experiments. ${ }^{\mathrm{a}} P<0.05,{ }^{\mathrm{b}} P<0.01$ versus the control group; ${ }^{\mathrm{c}} P<0.05$, ${ }^{\mathrm{d}} P<0.01$ versus the t-BHP-treated group; ${ }^{\mathrm{e}} \mathrm{P}<0.05,{ }^{\mathrm{f}} \mathrm{P}<0.01$ versus RSV $(10 \mu \mathrm{M})$ and t-BHP $(80 \mu \mathrm{M})$ co-treated group with control siRNA transfection

reaction, thereby leading to mitochondrial injury. The results suggested that $\mathrm{t}$-BHP reduced cell viability and increased apoptosis in endothelial cells, accompanied by depolarized $\Delta \psi \mathrm{m}$ and excessive $\mathrm{O}_{2}^{--}$generation in the mitochondria, which were notably attenuated by RSV treatment. These findings revealed an RSV-mediated pharmacologic preconditioning effect in endothelial cells.

Increased ROS from vessel tissue under pathological conditions is known to be the main cause of endothelial dysfunction. Evidence suggests that mitochondria are the primary source of ROS, and homeostatic regulation of mtROS is influenced by various enzymes involved in the tricarboxylic acid (TCA), ETC, OXPHOS and free-radical-scavenging systems in the mitochondria. ${ }^{23,24}$ The known site of mtROS production is the mitochondrial ETC, particularly complexes I and III, where $\mathrm{O}_{2}^{--}$is formed and subsequently reduced by dismutation to $\mathrm{H}_{2} \mathrm{O}_{2} \cdot \mathrm{H}_{2} \mathrm{O}$ can react with reduced $\mathrm{Fe}^{2+}$ and $\mathrm{Cu}^{2+}$ to produce highly toxic hydroxyl radicals $(\cdot \mathrm{OH})$ and can also penetrate membranes and leave the mitochondria. $\mathrm{O}_{2}^{--}, \mathrm{H}_{2} \mathrm{O}_{2}$ and $\cdot \mathrm{OH}$ are considered as the primary ROS. The mtROS scavenging system mainly includes SOD2, the glutathione and thioredoxin systems, which maintain a normal ratio of GSH/GSSG, NADPH/NADP ${ }^{+}$and NADH/NAD ${ }^{+}$. In the current study, RSV treatment resulted in an increased enzymatic activities of IDH2, GSH-Px and SOD2, which were required for scavenging excessive mtROS in mitochondria. Moreover, MitoSOX Red, a highly selective fluorescent probe for $\mathrm{O}_{2}^{--}$ within mitochondria, was applied to detect mtROS and the ROS generated in the mitochondria could thus be differentiated from the cytoplasmic and exogenous sources.

Sirtuins are $\mathrm{NAD}^{+}$-dependent enzymes that have been implicated in a wide range of physiological and pathophysiological conditions through deacetylation of numerous substrates. Sirtuins are important regulators of mammalian physiology and have different levels of $\mathrm{NAD}^{+}$-dependent protein deacetylase activity. ${ }^{25,26}$ Sirt3 has been shown to regulate mitochondrial metabolism by deacetylation of several enzymes involved in fatty acid oxidation, ketone body synthesis, TCA, OXPHOS, ROS generation and so on. Proteomics research has shown that several subunits of the enzymes in the mitochondrial ETC and TCA cycle have multiple acetylation modification sites, and increased acetylation was observed in Sirt3 ${ }^{-1-}$ tissues, indicating that the enzyme activities may be regulated by Sirt3. ${ }^{27-29}$ In addition, mice lacking Sirt3 showed striking hyperacetylation of mitochondrial proteins, which were associated with accelerated development of metabolic syndrome. ${ }^{30-32}$ Our current findings suggested that RSV promotes SOD2 deacetylation in mitochondria, which was suppressed by Sirt3 siRNA transfection, suggesting that Sirt3 plays a critical role in RSV-induced deacetylation in endothelial cells. SOD2 expression is believed to be an important cellular defense mechanism against to oxidative stress, whereas SOD2 deacetylation enhances mitochondrial scavenging capacity. ${ }^{33,34}$ 
Recent studies have revealed that Sirt3 is involved in mtDNA transcription. ${ }^{35}$ Sirt3 mediates FoxO3A binding to mtDNA, and transcription of mitochondrial-encoded core or catalytic subunits of the OXPHOS machinery increases respiration, which in turn decreases the byproducts of mitochondrial electron transfer reactions from the incomplete reduction of oxygen. The decrease of mitochondrial ETC efficiency and ATP synthesis affects mtROS homeostasis, and alterations in mitochondrial physiology can increase the ATP/ ROS ratio. A favorable balance in the ATP/ROS ratio may represent a common mechanism for extending the life span. Mitochondria in mammals undergoing caloric and dietary restrictions have been reported to show increased ATP/ROS ratios, in which cells generate the same amount of ATP with reduced oxidative stress and damage, with potential beneficial effects on life span. ${ }^{36}$ A similar positive effect on the ATP/ROS ratio was detected in adult flies, in which mitochondrial uncoupling in neurons was increased, and the life span extended. ${ }^{37}$ Interventions that maintain a beneficial mitochondrial ATP/ROS ratio could provide therapeutic opportunities for extending healthy life span and preventing disease. ${ }^{38}$ A previous study suggested that FoxO3A phosphorylation may be important for its mitochondrial import, and that once in the mitochondria, FoxO3A forms a complex with Sirt3 and mtRNAPol to activate transcription. ${ }^{35}$ As expected, RSV triggered the enrichment of Sirt3 in the mitochondria and activated the transcription of mtDNA-encoded OXPHOS units, which might be crucial for improving mitochondrial ETC efficiency (e.g., increased enzymatic activity of complex I) and reducing ROS generation, leading to sustained mtROS homeostasis. Our findings thus revealed a novel mechanism whereby RSV induction leads to an appropriate balance between mitochondrial energy production and mtROS generation, which is in turn responsible for the homeostatic regulation of redox status in HUVECs.

An expanding body of preclinical evidence suggests that RSV is associated with potential health benefits. Oral administration of RSV resulted in enhanced agonist-stimulated, endothelium-dependent relaxation in animal experiments $^{39}$ and human trials; ${ }^{40}$ however, the exact molecular mechanisms remain unknown. To investigate the mechanism of RSV-induced Sirt3 activation, we examined the activation of AMPK and PGC-1a, sensors of energy molecules. AMPK, which is known to act as a fuel gauge and a master switch regulating glucose and lipid metabolism, senses metabolic stress and integrates diverse physiological signals to restore the energy balance. ${ }^{41,42}$ It also serves as a regulator of cell survival or death in response to pathological stresses, such as hypoxia, osmotic stress and oxidative stress. ${ }^{43}$ AMPK thus plays a key role in intracellular metabolism and is an attractive therapeutic target, especially for energy-related diseases. ${ }^{44}$ In addition, fasting or nutrient excess may trigger the switching on/off of AMPK, which leads to alteration in PGC-1a activity. ${ }^{45}$ Furthermore, a previous report suggested that PGC-1a stimulated mouse Sirt3 activity in both muscle cells and hepatocytes, ${ }^{46}$ indicating that PGC- $1 a$ acts as an endogenous regulator of Sirt3. We therefore postulated that $\mathrm{RSV}$ regulates mtROS homeostasis through activation of the AMPK-PGC-1aSirt3 axis. As expected, our results demonstrated that RSV triggered AMPK phosphorylation and activation, which are required for PGC-1a expression. PGC-1a activated ERRa to cause ERRa coupling with ERRE in the Sirt3 promoter region, leading to Sirt3 mRNA transcription. These results are supported by those of Kong and colleagues. ${ }^{46}$ The AMPKPGC-1/a-Sirt3 signaling pathway could be a key route in the regulation of mtROS homeostasis by $\mathrm{RSV}$ treatment in endothelial cells.

HUVECs were used in the study, though there might be differences between the endothelial cells from arterial and venous system. ${ }^{4-49}$ The first and most important difference between the two is the arteriovenous oxygen difference. As well, the endothelial cells in arteries and veins might be exposed to other different physical conditions (e.g., shear stress). However, the extent to which mitochondrial physiology varies in the endothelium of different tissues is unknown, although certainly some mitochondrial functions, such as mtDNA repair ability, appear to differ even among pulmonary arterial, venous and microvascular endothelial cells. ${ }^{50}$ On the opposite, both of the arterial and venous mitochondria in endothelial cells share similar characteristics. Particularly, the endothelial mitochondria from the arteries and veins are both proposed as the central oxygen sensors of local environments. ${ }^{51}$ Furthermore, from the perspective of developmental biology, it was found that arterial and venous endothelial cells have molecularly defined identities that are evident before circulatory flow or even tubulogenesis. ${ }^{52}$ Although AS only occurs in arteries but never in veins (mainly attributed to the higher blood pressure in arteries), isolation of vascular endothelial cells from veins (such as HUVECs) is commonly applied in several in vitro studies. Indeed, endothelial cells can also be harvested from arterier tissues, such as from the large vessels by mechanical removal or collagenase digestion, ${ }^{53}$ or from human internal mammary arteries obtained from donors undergoing coronary artery bypass graft surgery. ${ }^{54}$ However, direct study of vascular endothelial function in humans is also difficult in part because of limited access to arteries. Therefore, access to peripheral veins is more practical and the umbilical vein has been used due to its wide availability. To our knowledge, use of cells collected from veins to study the endothelial cells is a widespread way at present, even if the arterial-venous differences in endothelial cells might be present.

In conclusion, the results of this study provide novel insights into the interpretation of the potential mechanisms responsible for the homeostatic regulation of mtROS levels by RSV in endothelial cells, in which Sirt3 plays a key role. These results may provide valuable clues in the search for new drugs that can be applied to endothelial protection.

\section{Materials and Methods}

Chemicals and antibodies. Cell culture media HyQ M199/EBSS (M199) and fetal bovine serum were provided from HyClone Laboratories (Logan, UT, USA). RSV, t-BHP, dimethylsulfoxide (DMSO) and compound $\mathrm{C}$ were purchased from Sigma-Aldrich (St. Louis, MO, USA). The 5,5,'6,6'-tetrachloro-1,1',3,3'tetraethylbenzimidazolylcarbocyanine iodide (JC-1), 5-aminoimidazole-4carboxamide 1- $\beta$-D-ribofuranoside (AICAR) and the antibody against $\beta$-actin were purchased from Beyotime Institute of Biotechnology (Beyotime Biotech, Beijing, China). A cell counting kit (CCK-8) was purchased from Dojindo Laboratories (Dojindo Molecular Technologies, Rockville, MD, USA). Antibodies against PGC-1 $\alpha$, Sirt3, Bcl-2, Bax, AIF and Cytc, mtRNAPol, FoxO3A, caspase 3, phosphorylated adenosine monophosphate-activated protein kinase (p-AMPK) 
and AMPK were obtained from Cell Signaling Technology (Beverly, MA, USA). MitoSOX Red mitochondrial superoxide indicator for live-cell imaging was obtained from Life Technologies (San Diego, CA, USA).
Cell culture. HUVECs were isolated from umbilical veins from fresh cords obtained at normal deliveries as reported before. ${ }^{55}$ The study protocol was approved by the Ethics Review Committee of Third Military Medical University.

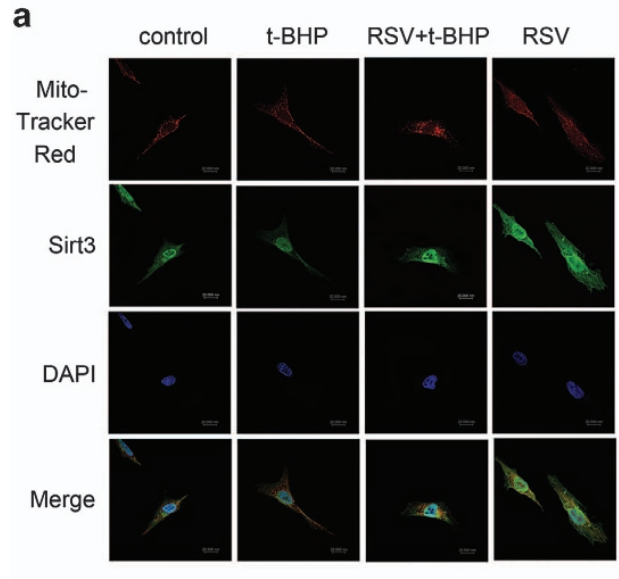

c

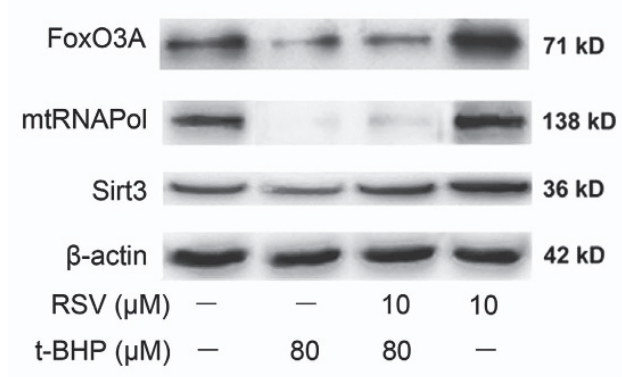

e

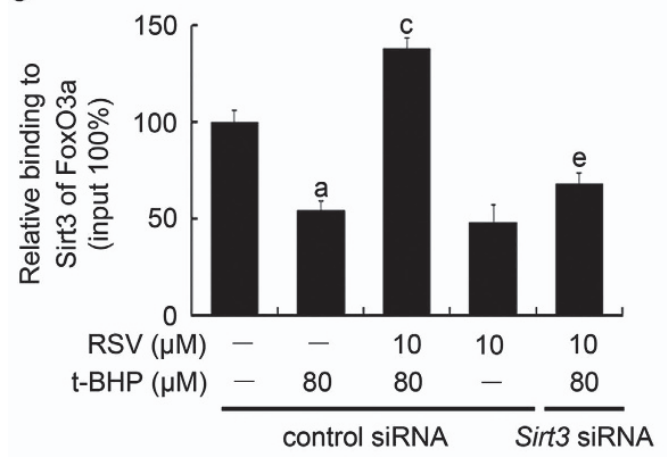

g

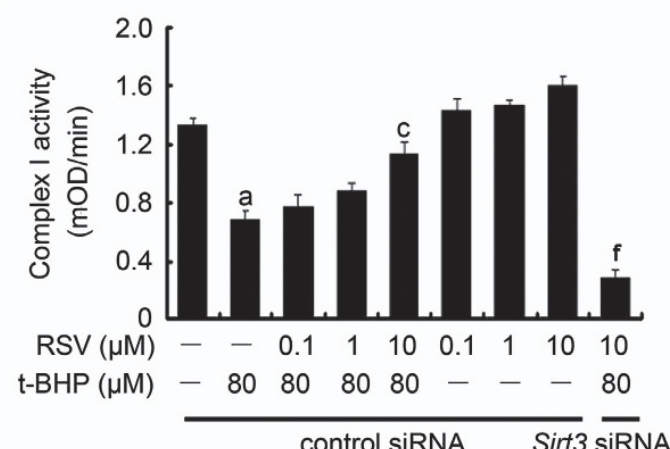

b

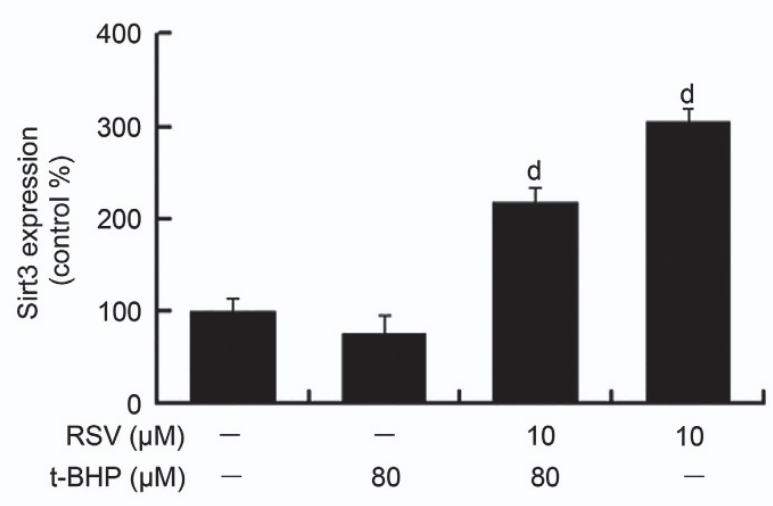

d

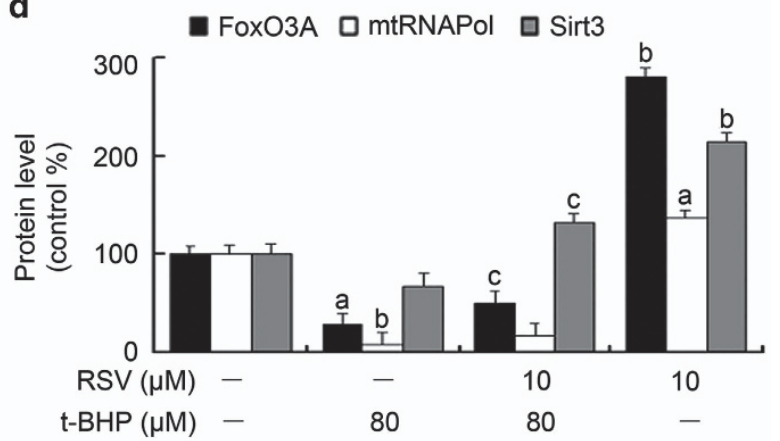

f

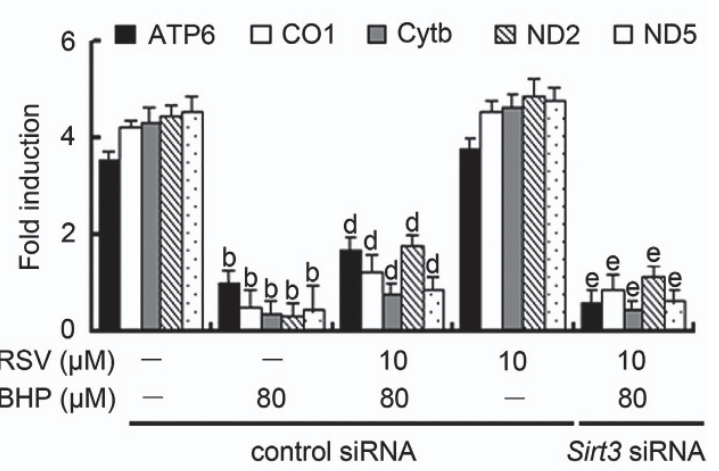

h

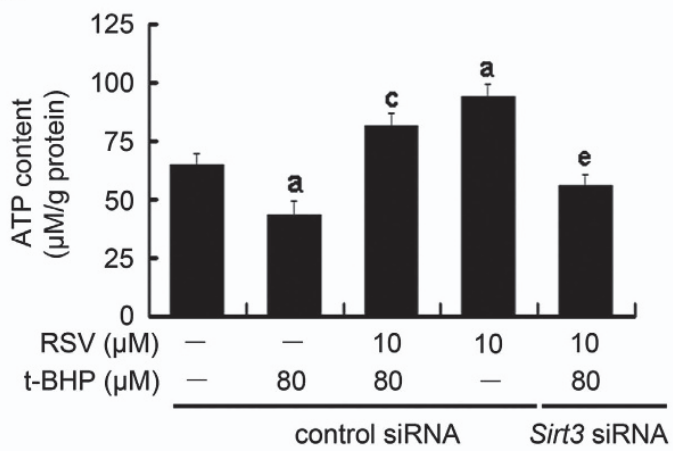


The cells purity was identified $>95 \%$ by immunofluorescence with specific anti-von Willebrand factor (VWF) antibody. HUVECs were cultured with M199 medium supplemented with $10 \%$ fetal bovine serum at $37^{\circ} \mathrm{C}$ in a humidified atmosphere containing $5 \% \mathrm{CO}_{2}$. All experiments were performed on the cells following 3-6 passages when the cells reached $80-90 \%$ confluence.

Cell viability assay. Cell viability was measured by the CCK- 8 assay according to the manufacturer's protocol. Briefly, cells were seeded in a 96-well microplate at a density of $8 \times 10^{3}$ cells per well and then treated with different doses $(20,30,40,50,60,70,80,90$ and $100 \mu \mathrm{M})$ of t-BHP for $4 \mathrm{~h}$. Meanwhile, cells were exposed to t-BHP of $80 \mu \mathrm{M}$ for different time intervals (1, 2, 4, 8, 12 and $24 \mathrm{~h}$ ). In addition, cells were preincubated with RSV for $2 \mathrm{~h}$ at a series of concentrations $(0.1$, $0.5,1,5,10$ and $15 \mu \mathrm{M}$, respectively). After removing the supernatant, cells were treated with or without $\mathrm{t}-\mathrm{BHP}$ of $80 \mu \mathrm{M}$ for an additional $4 \mathrm{~h}$. The control group was treated with $0.2 \%$ DMSO. Subsequently, CCK- 8 solution ( $10 \mu l /$ well) was added to each well and the plate was incubated at $37^{\circ} \mathrm{C}$ for $2 \mathrm{~h}$. The absorbance was measured using a monochromator microplate reader (Molecular Devices, Sunnyvale, CA, USA) at a wavelength of $450 \mathrm{~nm}$. The percentage of living cells was calculated by the ratio of the optical density of the experimental cells to that of the normal cells (set as $100 \%$ ).

Flow cytometric analysis. Cell apoptosis was detected with annexin-Vfluorescein isothiocyanate (FITC) apoptosis detection kit (Beyotime Biotech, Beijing, China) as described by the manufacture's instructions. Briefly, cells were pretreated with different concentrations of RSV $(0.1,1$ and $10 \mu \mathrm{M})$ for $2 \mathrm{~h}$, washed, and then treated with or without t-BHP $(80 \mu \mathrm{M})$ for another $4 \mathrm{~h}$. Thereafter, cells were collected and washed with ice-cold PBS twice, gently resuspended in the binding buffer and incubated with annexin V-FITC and propidium iodide (PI) for $15 \mathrm{~min}$ in dark. Cells were analyzed on a FACScan flow cytometer (Becton Dickinson, Franklin Lakes, NJ, USA). The total apoptosis rate of cells was calculated as the sum of the rates of cells observed in the lower-right quadrant and the upper-right quadrant.

Separation of cytosolic and mitochondrial fractions. The cytosolic and mitochondrial fractions were isolated as described. ${ }^{56}$ Briefly, $1 \times 10^{7}$ cells were harvested by centrifugation at $800 \times g$, washed in PBS and re-pelleted. Cells were digitonin-permeablilized for $5 \mathrm{~min}$ on ice at a density of $3 \times 10^{7} / \mathrm{ml}$ in cytosolic extraction buffer $\left(75 \mathrm{mM} \mathrm{NaCl}, 1 \mathrm{mM} \mathrm{NaH}_{2} \mathrm{PO}_{4}, 8 \mathrm{mM} \mathrm{Na}_{2} \mathrm{HPO}_{4}, 250 \mathrm{mM}\right.$ sucrose, $1 \mathrm{mM}$ phenylmethylsulfonyl fluoride (PMSF), $5 \mu \mathrm{g} / \mathrm{ml}$ leupeptin, $5 \mu \mathrm{g} / \mathrm{ml}$ aprotinin and $0.05 \%$ digitonin). Following the centrifugation step at $800 \times g$ at $4{ }^{\circ} \mathrm{C}$ for $10 \mathrm{~min}$, the supernatant was separated from the pellet comprising mitochondria and cellular debris. The supernatant containing cytoplasmic protein was further purified by centrifugation at $13000 \times g$ at $4{ }^{\circ} \mathrm{C}$ for $10 \mathrm{~min}$. The precipitated mitochondrial fractions were collected and quantified for the protein content $(3.5 \mathrm{mg} / \mathrm{ml})$. And then, the pellets were solubilized in the same volume of mitochondrial lysis buffer ( $50 \mathrm{mM}$ Tris $(\mathrm{pH} 7.4)$, $150 \mathrm{mM} \mathrm{NaCl}, 2 \mathrm{mM}$ EDTA, $2 \mathrm{mM}$ EGTA, 0.2\% Triton X-100, 0.3\% NP-40, $1 \mathrm{mM}$ PMSF, $5 \mu \mathrm{g} / \mathrm{ml}$ leupeptin and $5 \mu \mathrm{g} / \mathrm{ml}$ aprotinin). After centrifugation at $12000 \times \mathrm{g}$ at $4^{\circ} \mathrm{C}$ for $10 \mathrm{~min}$, supernatants were collected and used as the mitochondrial fraction, precipitation were collected and used as the cytoplasmic fraction.

Western blotting. Cells were collected by scraping with ice-cold PBS containing PMSF, and were lysed in the buffer. The protein concentration of the lysates was determined by Bradford method. Protein ( $80 \mathrm{mg}$ for each sample) was separated by sodium dodecyl sulfatepolyacrylamide gel (SDS-PAGE) and transferred to nitrocellulose membranes. After blocking (4\% non-fat milk), the membranes were probed with the indicated primary antibody overnight at $4{ }^{\circ} \mathrm{C}$, washed and incubated with a 1:6000 dilution of horseradish peroxidase (HRP)conjugated secondary antibody for $1 \mathrm{~h}$. The proteins were visualized by enhanced chemiluminescence (ECL; Amersham, UK) reagents for 1 min and exposed to CLXposure film (Pierce, Rockford, IL, USA). Finally, the blots were scanned, and densitometric analysis was performed on the scanning images using Scion ImageRelease Beta 4.02 software (Scion Corporation, Frederick, MD, USA).

RNA interference. The siRNAs for AMPK (human, sc-45312), PGC-1 $\alpha$ (human, sc-38884), estrogen-related receptor $\alpha$ (ERR $\alpha$; human, sc-44706) and Sirt3 (human, sc-61555) along with control siRNA (human, sc-44230) and siRNA transfection reagent (human, Sc-29528) were purchased from Santa Cruz Biotechnology (Santa Cruz, CA, USA). HUVECs were transfected with $100 \mathrm{nM}$ siRNA for 5-7 $\mathrm{h}$ according to the manufacturer's protocol. Then, cells were switched into fresh complete medium and incubated for an additional $24 \mathrm{~h}$. Thereafter, cells were harvested for further experiments.

Mitochondrial membrane potential $(\Delta \psi \mathrm{m})$ determination. Mitochondrial membrane potential $(\Delta y \mathrm{~m})$ was detected using the fluorescent, lipophilic dye, JC-1. JC-1 can easily penetrate cells with healthy mitochondria. A green fluorescent $\mathrm{JC}-1$ probe exists as a monomer at low membrane potentials; however, at higher potentials, JC-1 forms red-fluorescent 'J-aggregates'. The cells were incubated with $5 \mathrm{mg} / \mathrm{JC}-1$ for $1 \mathrm{~h}$, washed twice with PBS and resuspended in the serum-free medium. The fluorescence intensity was determined by confocal laser scanning microscope (CLSM; model TCS SP2; Leica Microsystems GmbH, Wetzlar, Germany). For simultaneous visualization of the green fluorescence from JC-1 monomer and the red fluorescence from JC-1 J-aggregates, a long pass filter system was used (excitation lines 488 and $561 \mathrm{~nm}$ ). Fixed exposure time and laser intensities were used during all acquisitions. The quantitative analysis of the red/ green fluorescence signal was detected and the built-in evaluation software (Leica LAS AF Lite, Mannheim, Germany) was used for calculation. The ratio of green (JC-1 monomers) and red fluorescence (JC-1 J-aggregates) were measured within randomly selected cells. In both the experimental and control groups 30 individual cells were measured in six identical cultures. All data were statistically evaluated using the Mann-Whitney test and the Kolmogorov-Smirnov test. The $\Delta y m$ was represented as the ratio of red to green fluorescence intensity.

Mitochondrial $\mathrm{O}_{2}^{--}$assessment. The mitochondrial $\mathrm{O}_{2}^{--}$generation was assessed using the MitoSOX Red (Invitrogen), a highly selective fluorescent probe for the detection of $\mathrm{O}_{2}^{--}$generated within mitochondria. HUVECs were added to glass coverslips at $8 \times 10^{3}$ cells per well and were treated as indicated. MitoSOX Red reagent stock solution was diluted with $\mathrm{HBSS} / \mathrm{Ca} / \mathrm{Mg}$ buffer to make a working solution of $5 \mu \mathrm{M}$. Then, cells were incubated with $5 \mu \mathrm{M}$ MitoSOX Red reagent in dark at $37^{\circ} \mathrm{C}$ for $10 \mathrm{~min}$. Cells were washed gently three times with warm buffer and were stained with counterstains as desired amount for imaging. Fluorescence intensities of mtROS were determined using an Infinite M200 microplate reader (Tecan Group Ltd., Männedorf, Switzerland) and images were visualized by a CLSM (model TCS SP2; Leica Microsystems $\mathrm{GmbH}$ ) with the emission wavelength of $579 \mathrm{~nm}$ and the excitation wavelength of $510 \mathrm{~nm}$, respectively.

Mitochondrial enzyme activities. The enzyme activities of complex I, IDH2, SOD2 and GSH-Px were assessed in mitochondrial homogenates, which

Figure 5 RSV reduced mtROS generation through the promotion of Sirt3-mediated mtDNA transcription. Cells were pretreated by RSV ( $10 \mu \mathrm{M})$ for $2 \mathrm{~h}$, then washed, and followed by treatment with or without t-BHP $(80 \mu \mathrm{M})$ for an additional $4 \mathrm{~h}$. (a) Representative images show Sirt3 expression within mitochondria in HUVECs by immuofluorescence assay. Red fluorescence indicates mitochondria labeled with Mito-tracker Red. Green fluorescence originates from anti-Sirt3 labeling. And blue fluorescence indicates nuclei stained with DAPI. (b) The bar graph shows quantification of Sirt3 expression in the mitochondria. (c) The expressions of mtRNAPol, FoxO3A and Sirt3 in the mitochondria were determined by western blot. (d) The bar graph shows quantification of the indicated proteins. Sirt3 was knocked down by Sirt3 siRNA transfection as described in the Materials and Methods section. The cells were pretreated with RSV of $10 \mu \mathrm{M}$ for $2 \mathrm{~h}$, then washed, and followed by incubation with fresh medium in the presence or absence of t-BHP of $80 \mu \mathrm{M}$ for an additional $4 \mathrm{~h}$. (e) Cells were harvested for ChIP analysis and the recruitment of FoxO3A was measured by real-time PCR assay using primers targeted to Sirt3. The results were plotted relative to those of the total input controls (untreated chromatin). (f) The expressions of mtDNA-encoded genes of ATP6, CO1, Cytb, ND2 and ND5 along with the housekeeping genes of $\beta$-actin, GAPDH and 18s rRNA were determined by real-time PCR assay. In order to generate a relative expression ratio, the expressions of the genes of interest were normalized to the geometric average of the housekeeping genes. (g) The enzyme activity of complex I was determined using the corresponding assay kit, according to the manufacturer's instructions. (h) ATP content was determined using an ATP determination kit. All results are presented as means \pm S.E.M. of at least three independent experiments. ${ }^{\mathrm{a}} P<0.05,{ }^{\mathrm{b}} \mathrm{P}<0.01$ versus the control group; ${ }^{\mathrm{C}} \mathrm{P}<0.05,{ }^{\mathrm{d}} \mathrm{P}<0.01$ versus the t-BHP-treated group; ${ }^{\mathrm{e}} P<0.05,{ }^{\mathrm{f}} P<0.01$ versus RSV (10 $\left.\mu \mathrm{M}\right)$ and $\mathrm{t}-\mathrm{BHP}(80 \mu \mathrm{M})$ co-treated group with control siRNA transfection 
contained protease inhibitors only. The complex I activity was measured with complex I enzyme activity microplate assay kit (Abcam, Cambridge, UK) and its activity was determined from the oxidation of reduced nicotinamide adenine dinucleotide (NADH/NAD ${ }^{+}$) to $\mathrm{NAD}^{+}$. The IDH2 activity was assayed by IDH activity assay kit (Sigma-Aldrich) with a temperature-controlled spectrofluorometer (Hitachi
F-4500, Tokyo, Japan) at the excitation wavelength of $366 \mathrm{~nm}$ and emission wavelength of $450 \mathrm{~nm}$. And the activities of SOD2 and GSH-Px in the mitochondria were determined by commercial assay kits purchased from Nanjing Jiancheng Bioengineering Institute (Nanjing, Jiangsu, China), according to the manufacturer's instructions, respectively. a

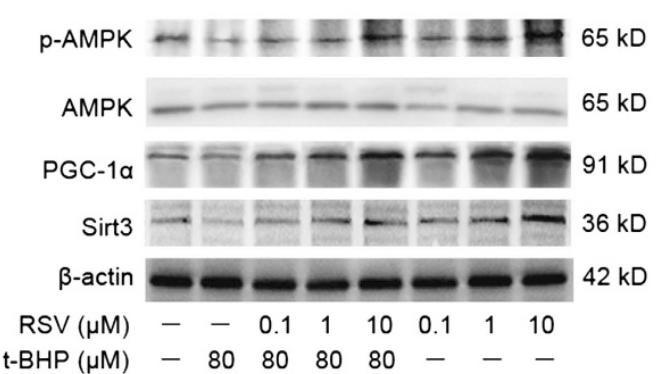

C

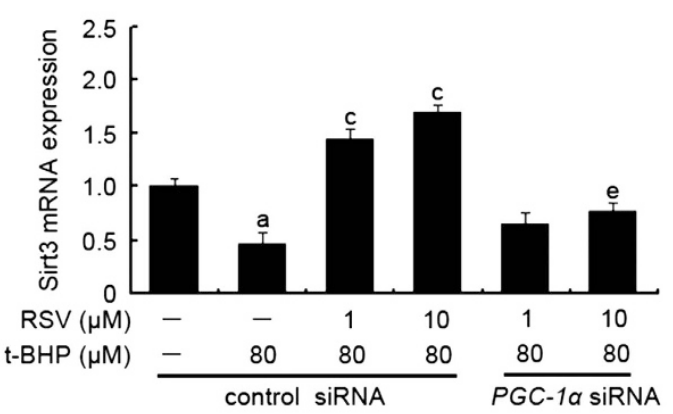

e

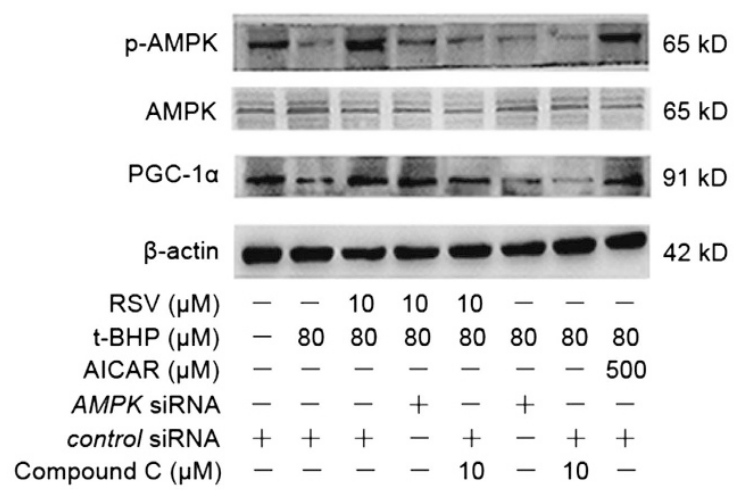

g

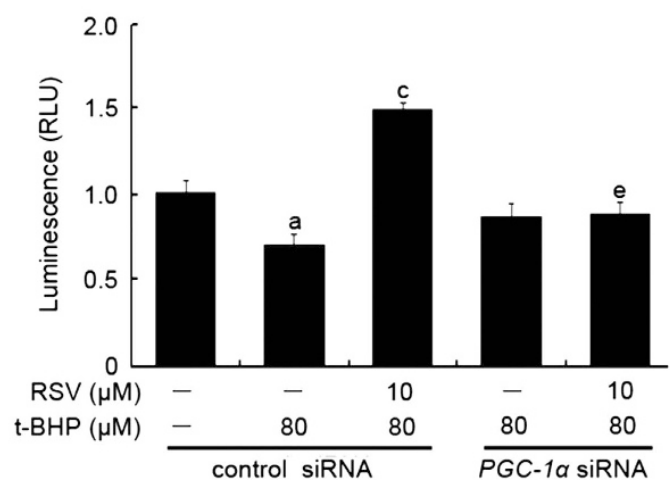

b

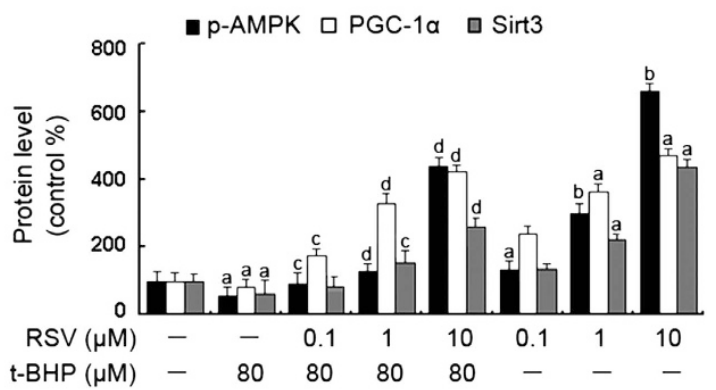

d

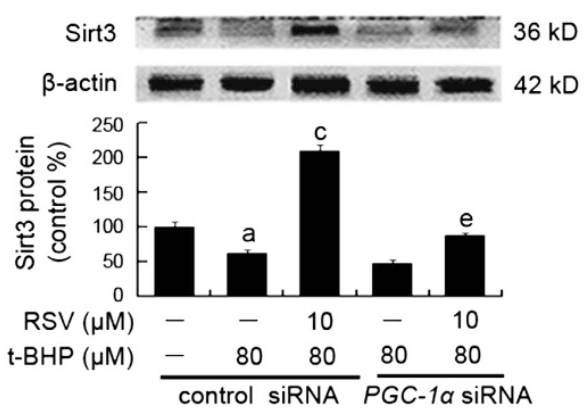

$\mathbf{f}$

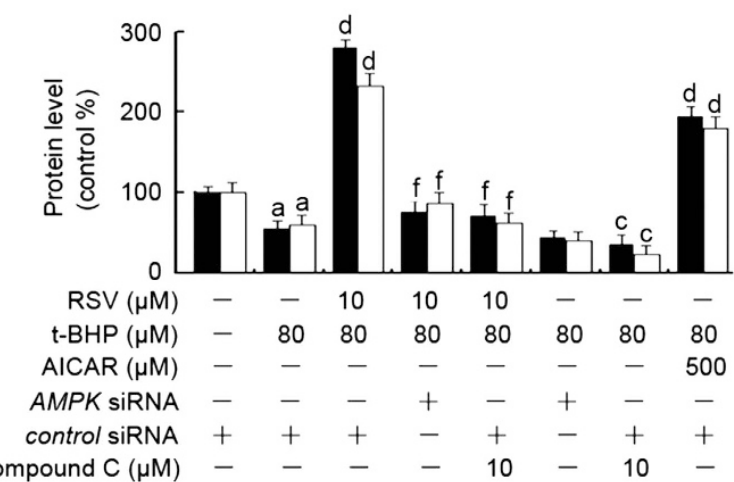

h

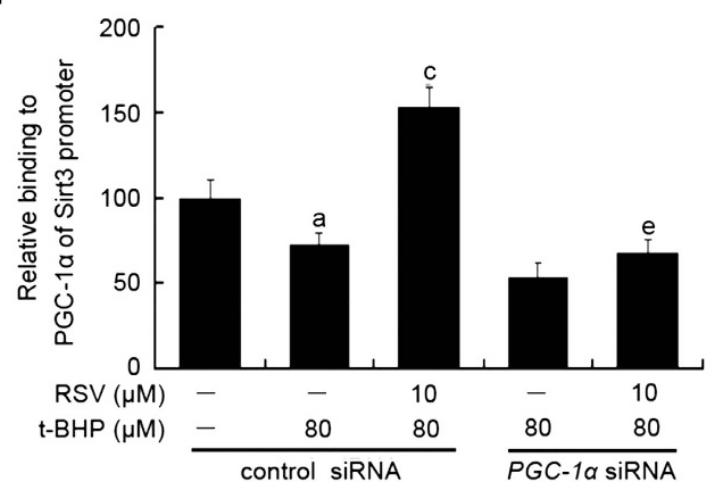


Immunoprecipitation of acetyl lysine-modified SOD2. Mitochondrial proteins $(150 \mu \mathrm{g})$ were incubated at $4^{\circ} \mathrm{C}$ with $5 \mu \mathrm{g}$ of antibody against acetyl lysine (K68) SOD2 (ac-SOD2; Cambridge, MA, USA) in a total volume of $250 \mu \mathrm{l}$ for $24 \mathrm{~h}$. Following addition of protein A/G-agarose (50 $\mu$; Santa Cruz Biotechnology) the solution was incubated for $2 \mathrm{~h}$ at $4{ }^{\circ} \mathrm{C}$, centrifuged and the agarose complexes extensively washed with Tris-buffered saline containing $0.05 \%(\mathrm{v} / \mathrm{V})$ Tween 20 (Sigma-Aldrich). Pellets were stored at $-20^{\circ} \mathrm{C}$ until further analysis by SDS-PAGE.

Immunofluorescence imaging. Confluent HUVECs were grown on glass coverslips and then harvested. The slides were incubated with the Mito-Tracker Red (Invitrogen) of $80 \mathrm{nM}$ for $20 \mathrm{~min}$, and then were fixed with $4 \%(\mathrm{v} / \mathrm{v})$ paraformaldehyde for $20 \mathrm{~min}$ at $37^{\circ} \mathrm{C}$ and permeabilized with $0.1 \%$ Triton X-100 for $10 \mathrm{~min}$ at room temperature. The slides were blocked with PBS supplemented with $0.1 \%(\mathrm{w} / \mathrm{v}) \mathrm{BSA}$ and $0.1 \%(\mathrm{w} / \mathrm{v})$ Tween 20 , thereafter, incubated with antiSirt3 antibody ( $1: 50$ dilution) overnight at $4{ }^{\circ} \mathrm{C}$. After washing with PBS, the slides were incubated for $1 \mathrm{~h}$ at room temperature with a FITC-conjugated secondary antibody (1:1000 dilution; Molecular Probes, Eugene, OR, USA). Finally, the slides were washed twice with PBS and the nuclei were counterstained with 4', 6'-diamidino-2-phenylindole (DAPI) for $5 \mathrm{~min}$ at room temperature. Images of the stained cells were obtained using CLSM (model TCS SP2; Leica Microsystems $\mathrm{GmbH}$ ) and the intracellular fluorescence intensity was expressed as the percentage relative to the control group (set as $100 \%$ ), respectively.

Real-time PCR. Total cellular RNA was extracted by Trizol RNA isolation kit (Invitrogen) according to the manufacture's protocol. Then, cDNA was synthesized from $1 \mu \mathrm{g}$ RNA for each sample with random primers according to the protocol of Invitrogen. Quantitative real-time PCR was performed on the iQ5 multicolor realtime PCR detection system (Bio-Rad, Hertfordshire, UK) with specific primers (Table 1). All primers were designed by the software Primer Premier 5.0 and synthesized by Invitrogen (Shanghai, China). In the reaction, $1 \mu \mathrm{l}$ cDNA of each sample was mixed with SYBR Green JumpStart Taq ReadyMix (Fisher Scientific,

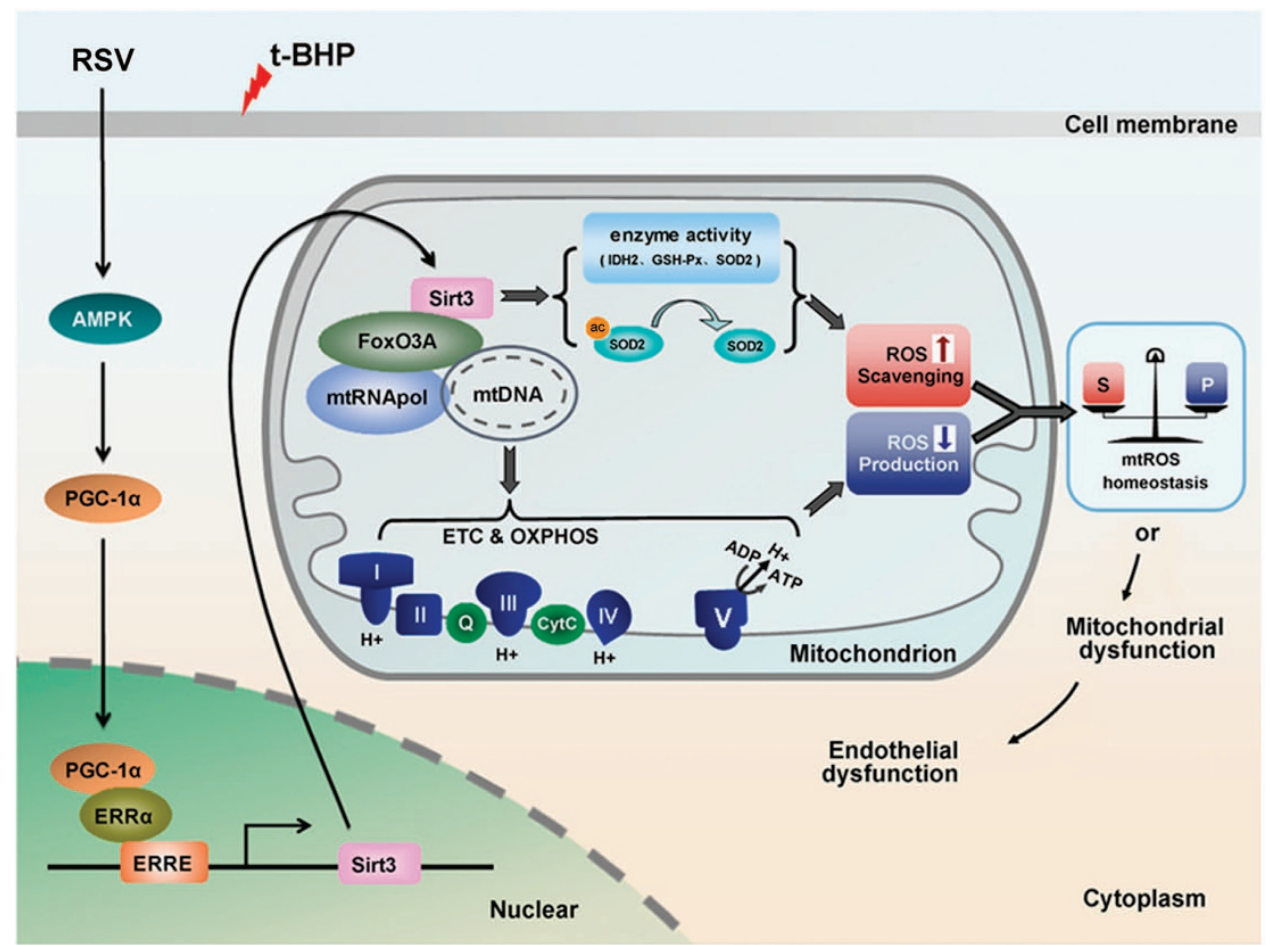

Figure 7 Proposed mechanism for RSV in the regulation of mtROS homeostasis through Sirt3 signaling pathway in HUVECs. RSV triggers AMPK-PGC-1 $\alpha$ signaling pathway, which is required for ERR $\alpha$-dependent Sirt3 transcription and subsequent enrichment within the mitochondria, thereby leading to deacetylation and activation of mitochondrial enzymes involved in mtROS regulation as well as stimulation of mitochondrial ETC efficiency by upregulation of mtDNA-encoded gene expression and ATP synthesis, finally contributing to mtROS homeostasis in HUVECs. I, complex I; II, complex II; III, complex III; IV, complex IV; V, ATP synthase enzymes; Q, CoQ; Cytc, cytochrome $c$

Figure 6 RSV stimulated AMPK-PGC-1 $\alpha$-ERR $\alpha$-Sirt3 signaling pathway in HUVECs. (a) Cells were pretreated with RSV $(0.1,1$ and $10 \mu \mathrm{M})$ for $2 \mathrm{~h}$ and then treated with or without t-BHP of $80 \mu \mathrm{M}$ for an additional $4 \mathrm{~h}$. The cells were harvested and lysed to detect protein levels of AMPK, p-AMPK, PGC-1 $\alpha$ and Sirt3 by western blot assay. (b) The bar charts show quantification of the indicated proteins. Cells were transfected with PGC-1 $\alpha$ siRNA as described in the Materials and Methods section. (c) The mRNA levels of the Sirt3 genes were determined by real-time PCR assay. (d) The cells were collected and lysed, and Sirt3 protein levels were detected by western blot analysis. Cells were preincubated with compound C $(10 \mu \mathrm{M})$, AMPK siRNA or AICAR $(500 \mu \mathrm{M})$ as described in the Materials and Methods section. Then cells were pretreated with RSV of $10 \mu \mathrm{M}$ for $2 \mathrm{~h}$, followed by incubation in the presence or absence of t-BHP of $80 \mu \mathrm{M}$ for an additional $4 \mathrm{~h}$. (e) The expressions of AMPK, p-AMPK and PGC-1 $\alpha$ were measured by western blot. (f) The bar charts show quantification of the indicated proteins. Cells were transfencted with PGC-1 $\alpha$ siRNA as described in the Materials and Methods section. At 24-h posttransfection, the cells were pretreated with RSV of $10 \mu \mathrm{M}$ for $2 \mathrm{~h}$ and then treated with or without t-BHP of $80 \mu \mathrm{M}$ for an additional $4 \mathrm{~h}$. (g) Cells were harvested using ChIP assay, and real-time PCR assay was carried out using primers specific to the Sirt3 promoter. The results were determined relative to those of the total input controls (untreated chromatin). (h) Cells were co-transfected with pRL-TK reporter plasmid supplemented with ERRE-luc or NEG-PG04 (vehicle control), respectively. ERR $\alpha$ was knocked down by ERR $\alpha$ siRNA transfection, as described in the Materials and Methods section. And then cells were pretreated with RSV of $10 \mu \mathrm{M}$ for $2 \mathrm{~h}$, followed by treatment with or without $\mathrm{t}$-BHP of $80 \mu \mathrm{M}$ for an additional $4 \mathrm{~h}$. Both firefly and Renilla luciferase activities were measured sequentially using the Dual-Glo luciferase reporter assay system. All results are presented as means \pm S.E.M. of at least three independent experiments. ${ }^{\mathrm{a}} P<0.05,{ }^{\mathrm{b}} P<0.01$ versus the control group; ${ }^{\mathrm{c}} P<0.05,{ }^{\mathrm{d}} P<0.01$ versus $\mathrm{t}-\mathrm{BHP}$-treated group; ${ }^{\mathrm{e}} \mathrm{P}<0.05,{ }^{\mathrm{f}} \mathrm{P}<0.01$ versus RSV $(10 \mu \mathrm{M})$ and t-BHP $(80 \mu \mathrm{M})$ co-treated group with control siRNA transfection 
Table 1 Primer details

\begin{tabular}{|c|c|c|c|}
\hline Primer & Forward $\left(5^{\prime}-3^{\prime}\right)$ & Reverse $\left(5^{\prime}-3^{\prime}\right)$ & Annealing temperatures $\left({ }^{\circ} \mathrm{C}\right)$ \\
\hline $\begin{array}{l}\text { Atp } 6 \\
\text { COX } 1 \\
\text { ND2 } \\
\text { ND5 } \\
\text { Cyto b } \\
\text { Sirt3 } \\
\text { GAPDH } \\
\text { B-actin } \\
\text { 18s rRNA }\end{array}$ & $\begin{array}{l}\text { GCCGCAGTACTGATCATTCTATTTC' } \\
\text { CCCCGCATAAACAACATAAGC } \\
\text { AAACCCTCGTTCCACAGAAGC' } \\
\text { GCAGCCATTCAAGCAATCCTA } \\
\text { TCGGCATTATCCTCCTGCTT } \\
\text { CCCAGTGGCATTCCAGACTT } \\
\text { CAACTTTGGTATCGTGGAAGGAC } \\
\text { CTGGCACCCAGCACAATG } \\
\text { GAGTATGGTTGCAAAGCTGAAACTT }\end{array}$ & $\begin{array}{l}\text { TCGGTTGTTGATGAGATATTTGGA } \\
\text { CAGATGCGAGCAGGAGTAGGA } \\
\text { GGATTATGGATGCGGTTGCT } \\
\text { AGGCGAGGATGAAACCGATA } \\
\text { CTCACGGGAGGACATAGCCTCT } \\
\text { AAGGGCTTGGGGTTGTGAAA } \\
\text { ACAGTCTTCTGGGTGGCAGTG } \\
\text { GTTCATGAGGCACACCTAGCC } \\
\text { TGAGTCAAATTAAGCCGCAGG }\end{array}$ & $\begin{array}{l}55 \\
57 \\
58 \\
60 \\
51 \\
55 \\
60 \\
55 \\
58\end{array}$ \\
\hline
\end{tabular}

Atlanta, GA, USA) according to the manufacture's instructions. And the PCR conditions: denaturation step of $5 \mathrm{~min}$ at $95^{\circ} \mathrm{C}$; amplification for 40 cycles (denaturation: $30 \mathrm{~s}$ at $95^{\circ} \mathrm{C}$; annealing: $30 \mathrm{~s}$ at corresponding temperature; extension: $30 \mathrm{~s}$ at $72^{\circ} \mathrm{C}$ ); final extension for $7 \mathrm{~min}$ at $72^{\circ} \mathrm{C}$. All the samples have three parallel wells and the experiments were repeated at least three times. The melt-curve analysis was used to confirm specific product formation. Housekeeping genes used in this study were $\beta$-actin, glyceraldehyde-3-phosphate dehydrogenase (GAPDH) and 18s ribosomal RNA (18s rRNA). In order to generate a relative expression ratio, the mRNA level of the gene of interest was normalized to the geometric average mRNA level of the housekeeping genes.

Chromatin immunoprecipitation analysis of genomic and mtDNA. ChIP analysis for genomic DNA and mtDNA was performed with Pierce Agarose ChIP kit as described previously with minor modifications. ${ }^{57}$ Briefly, formaldehyde was added directly to tissue culture medium to a final concentration of $1 \%$. Cross-linking was allowed to proceed for $15 \mathrm{~min}$ at room temperature and then stopped by the addition of glycine to a final concentration of $0.125 \mathrm{M}$. Cross-linked cells were scraped, washed with PBS, and then lysed in CLB (10 mM Tris ( $\mathrm{pH} 8.0)$, $10 \mathrm{mM} \mathrm{NaCl}$ and $0.2 \% \mathrm{NP}-40$ ) with protease inhibitors ( $1 \mathrm{mM}$ phenylmethylsulphonylfluoride, $5 \mathrm{mM} \mathrm{NaF}, 1 \mathrm{mM} \mathrm{Na}_{3} \mathrm{VO}_{4}, 1,5 \mu \mathrm{M}$ pepstatin, $2 \mu \mathrm{M}$ leupeptin, $10 \mu \mathrm{g} / \mathrm{ml}$ aprotinin, $1 \mathrm{mM} \mathrm{NaB}$ and $40 \mu \mathrm{M}$ bestatin). The mitochondrial fraction was enriched and lysed by mitochondrial lysis buffer (50 mM Tris-Cl pH 8.1, $10 \mathrm{mM}$ EDTA and $1 \%$ SDS) or purified using the Qproteome mitochondria isolation kit (Qiagen, Valencia, CA, USA). The chromatin solution was sonicated, cleared by centrifugation and the supernatant was collected; $1 \%$ of the supernatant was taken as input. Five micrograms of antibody per point was used to immunoprecipitate chromatin-bound complexes. Immunoprecipitation was performed on a rotating platform overnight at $4{ }^{\circ} \mathrm{C}$. Antibodies against PGC- $1 \alpha$ (for genomic DNA) and Sirt3 (for $\mathrm{mtDNA}$ ) were used for ChIP analysis, respectively. And the monoclonal $\lg G$ was used as unrelated antibodies. Immunocomplexes were pulled down using protein G (GE Healthcare, Milwaukee, WI, USA). Following extensive washing, bound DNA was reverse cross-linked and purified, then analyzed by real-time PCR assay as described previously. ${ }^{44}$ The primers for Sirt3 promotor was 5'-AGTAAGACCCGAGGTAGGAG-3' (forward) and 5'-GGAATAAGAGGGATGA CAGA-3' (reverse).

Adenosine triphosphate quantification. HUVECs were grown on glass coverslips at a density of $1.0 \times 10^{6}$ cells per well and then harvested. Cells were treated as indicated, then ATP content was measured using an ATP determination kit (Invitrogen, Carlsbad, CA, USA) according to the manufacturer's instructions. Briefly, the cells were washed twice in PBS and lysed with $500 \mu$ l of $0.2 \mathrm{M} \mathrm{HCLO}_{4}$ and centrifuged at $5000 \times g$ for $10 \mathrm{~min}$. The supernatant was mixed with the ATP determination kit reaction buffer containing luciferin and luciferase. After gentle mixing, the readings for the above mixtures were taken with the luminometer (Turner Designs, Sunnyvale, CA, USA). ATP contents were calculated by using an ATP standard curve.

Luciferase assay. HUVECs $\left(1 \times 10^{5}\right.$ cells per well) were maintained in 24-well plates until $60-70 \%$ confluence. Transient transfection was preformed by Lipofectamine 2000 (Invitrogen) transfection reagent according to the manufacturer's protocol. Briefly, cells were co-transfected with $0.2 \mu \mathrm{g} /$ well of estrogen receptor response element (ERRE)-luc plasmid HPRM24033-PG04 (Promega, Madison, WI, USA; Supplementary Figure 1A) and pRL-TK reporter plasmid (Promega; Supplementary Figure $1 \mathrm{~B}$ ), respectively. The mass of transfected plasmids was balanced with empty vector NEG-PG04 (Promega; Supplementary Figure 1C).
After transfection for $48 \mathrm{~h}$, cells were treated with RSV $(10 \mu \mathrm{M})$ for $2 \mathrm{~h}$ and followed by t-BHP $(80 \mu \mathrm{M})$ treatment for an additional $4 \mathrm{~h}$. Thereafter, cells were lysed and both of the firefly and Renilla luciferase activities were measured sequentially with the Dual-Glo luciferase reporter assay system (Promega) according to the manufacturer's instructions.

Statistical analysis. All the experiments were performed at least three times and results are expressed as the mean \pm S.E.M. Raw data were analyzed with SPSS 13.0 software (Chicago, IL, USA). Statistical analysis for multiple group comparisons was performed by one-way analysis of variance, followed by post hoc least significant difference tests. A two-sided $P$-value $<0.05$ was considered statistically significant.

\section{Conflict of Interest}

The authors declare no conflict of interest.

Acknowledgements. This work was supported by the National Natural Science Foundation of China (grant number 81172670) and the Youth Innovation Foundation of the Third Military Medical University (2012XJQ06).

1. Goldschmidt-Clermont PJ, Dong C, Seo DM, Velazquez OC. Atherosclerosis, inflammation, genetics, and stem cells: 2012 update. Curr Atheroscler Rep 2012; 14: 201-210.

2. Forstermann U. Oxidative stress in vascular disease: causes, defense mechanisms and potential therapies. Nat Clin Pract Cardiovasc Med 2008; 5: 338-349.

3. Jang C, Arany Z. Metabolism: sweet enticements to move. Nature 2013; 500: 409-411.

4. Dromparis $\mathrm{P}$, Michelakis ED. Mitochondria in vascular health and disease. Annu Rev Physiol 2013; 75: 95-126.

5. Kluge MA, Fetterman JL, Vita JA. Mitochondria and endothelial function. Circ Res 2013; 112 : 1171-1188.

6. Tang X, Luo YX, Chen HZ, Liu DP. Mitochondria, endothelial cell function, and vascular diseases. Front Physiol 2014; 5: 175.

7. Borriello A, Cucciolla V, Della Ragione F, Galletti P. Dietary polyphenols: focus on resveratrol, a promising agent in the prevention of cardiovascular diseases and control of glucose homeostasis. Nutr Metab Cardiovasc Dis 2010; 20: 618-625.

8. Pallauf K, Giller K, Huebbe P, Rimbach G. Nutrition and healthy ageing: calorie restriction or polyphenol-rich 'MediterrAsian' diet? Oxid Med Cell Longev 2013; 2013: 707421.

9. Agarwal B, Campen MJ, Channell MM, Wherry SJ, Varamini B, Davis JG et al. Resveratrol for primary prevention of atherosclerosis: clinical trial evidence for improved gene expression in vascular endothelium. Int J Cardiol 2013; 166: 246-248.

10. Chen $M-I, Y i$, Jin X, Xie $Q$, Zhang T, Zhou X et al. Absorption of resveratrol by vascular endothelial cells through passive diffusion and an SGLT1-mediated pathway. J Nutr Biochem 2013; 24: 1823-1829.

11. Chen ML, Yi L, Jin X, Liang XY, Zhou Y, Zhang T et al. Resveratrol attenuates vascular endothelial inflammation by inducing autophagy through the cAMP signaling pathway. Autophagy 2013; 9: 2033-2045.

12. Tseng AH, Shieh SS, Wang DL. SIRT3 deacetylates FOXO3 to protect mitochondria against oxidative damage. Free Radic Biol Med 2013; 63: 222-234.

13. Aneiros $E$, Dabrowski M. Novel temperature activation cell-based assay on thermo-TRP ion channels. J Biomol Screen 2009; 14: 662-667.

14. Peserico A, Chiacchiera F, Grossi V, Matrone A, Latorre D, Simonatto M et al. A novel AMPK-dependent FoxO3A-SIRT3 intramitochondrial complex sensing glucose levels. Cell Mol Life Sci 2013; 70: 2015-2029.

15. Kong X, Wang R, Xue Y, Liu X, Zhang H, Chen $Y$ et al. Sirtuin 3, a new target of PGC-1alpha, plays an important role in the suppression of ROS and mitochondrial biogenesis. PLoS One 2010; 5: e11707. 
16. Wan Z, Root-McCaig J, Castellani L, Kemp BE, Steinberg GR, Wright DC. Evidence for the role of AMPK in regulating PGC-1 alpha expression and mitochondrial proteins in mouse epididymal adipose tissue. Obesity 2014; 22: 730-738.

17. Desquiret-Dumas V, Gueguen N, Leman G, Baron S, Nivet-Antoine V, Chupin S et al. Resveratrol induces a mitochondrial complex l-dependent increase in NADH oxidation responsible for sirtuin activation in liver cells. J Biol Chem 2013; 288: 36662-36675.

18. Melemedjian OK, Asiedu MN, Tillu DV, Sanoja R, Yan J, Lark A et al. Targeting adenosine monophosphate-activated protein kinase (AMPK) in preclinical models reveals a potential mechanism for the treatment of neuropathic pain. Mol Pain 2011; 7: 70.

19. Chowdhury SK, Dobrowsky RT, Fernyhough P. Nutrient excess and altered mitochondria proteome and function contribute to neurodegeneration in diabetes. Mitochondrion 2011; 11 : 845-854.

20. Hirschey MD, Shimazu T, Jing E, Grueter CA, Collins AM, Aouizerat B et al. SIRT3 deficiency and mitochondrial protein hyperacetylation accelerate the development of the metabolic syndrome. Mol Cell 2011; 44: 177-190.

21. Chen CY, Yi L, Jin X, Mi MT, Zhang T, Ling WH et al. Delphinidin attenuates stress injury induced by oxidized low-density lipoprotein in human umbilical vein endothelial cells. Chem Biol Interact 2010; 183: 105-112.

22. Liu WH, Kao PH, Chiou YL, Lin SR, Wu MJ, Chang LS. Catalytic activity-independent pathway is involved in phospholipase A(2)-induced apoptotic death of human leukemia U937 cells via $\mathrm{Ca}(2+)$-mediated p38 MAPK activation and mitochondrial depolarization. Toxicol Lett 2009; 185: 102-109.

23. Kai L, Samuel SK, Levenson AS. Resveratrol enhances p53 acetylation and apoptosis in prostate cancer by inhibiting MTA1/NuRD complex. Int $J$ Cancer 2010; 126 : 1538-1548.

24. Danz ED, Skramsted J, Henry N, Bennett JA, Keller RS. Resveratrol prevents doxorubicin cardiotoxicity through mitochondrial stabilization and the Sirt1 pathway. Free Radic Biol Med 2009; 46: 1589-1597.

25. Jacobs KM, Pennington JD, Bisht KS, Aykin-Burns N, Kim HS, Mishra M et al. SIRT3 interacts with the daf-16 homolog $\mathrm{FOXO} 3 \mathrm{a}$ in the mitochondria, as well as increases FOXO3a dependent gene expression. Int J Biol Sci 2008; 4: 291-299.

26. Kim HS, Patel K, Muldoon-Jacobs K, Bisht KS, Aykin-Burns N, Pennington JD et al. SIRT3 is a mitochondria-localized tumor suppressor required for maintenance of mitochondrial integrity and metabolism during stress. Cancer Cell 2010; 17: 41-52.

27. Chen IC, Chiang WF, Chen PF, Chiang HC. STRESS-responsive deacetylase SIRT3 is up-regulated by areca nut extract-induced oxidative stress in human oral keratinocytes. J Cell Biochem 2014; 115: 328-339.

28. Dufour CR, Wilson BJ, Huss JM, Kelly DP, Alaynick WA, Downes M et al. Genome-wide orchestration of cardiac functions by the orphan nuclear receptors ERRalpha and gamma. Cell Metab 2007; 5: 345-356.

29. Balaban RS, Nemoto S, Finkel T. Mitochondria, oxidants, and aging. Cell 2005; 120 483-495.

30. Hirschey MD, Shimazu T, Goetzman E, Jing E, Schwer B, Lombard DB et al. SIRT3 regulates mitochondrial fatty-acid oxidation by reversible enzyme deacetylation. Nature 2010; 464: 121-125

31. Cimen H, Han MJ, Yang Y, Tong Q, Koc H, Koc EC. Regulation of succinate dehydrogenase activity by SIRT3 in mammalian mitochondria. Biochemistry 2010; 49: 304-311.

32. Brand MD. The sites and topology of mitochondrial superoxide production. Exp Gerontol 2010; 45: 466-472

33. Haigis MC, Sinclair DA. Mammalian sirtuins: biological insights and disease relevance. Annu Rev Pathol 2010; 5: 253-295.

34. Finkel T, Deng CX, Mostoslavsky R. Recent progress in the biology and physiology of sirtuins. Nature 2009; 460: 587-591.

35. Choudhary C, Kumar C, Gnad F, Nielsen ML, Rehman M, Walther TC et al. Lysine acetylation targets protein complexes and co-regulates major cellular functions. Science 2009; 325: 834-840.

36. Kim SC, Sprung $R$, Chen $Y, X u Y$, Ball $H$, Pei $J$ et al. Substrate and functiona diversity of lysine acetylation revealed by a proteomics survey. Mol Cell 2006; 23 : 607-618.

37. Schwer B, Eckersdorff M, Li Y, Silva JC, Fermin D, Kurtev MV et al. Calorie restriction alters mitochondrial protein acetylation. Aging Cell 2009; 8: 604-606
38. Lombard DB, Alt FW, Cheng HL, Bunkenborg J, Streeper RS, Mostoslavsky R et al. Mammalian Sir2 homolog SIRT3 regulates global mitochondrial lysine acetylation. Mol Cell Biol 2007; 27: 8807-8814.

39. Yoshino J, Imai S. Mitochondrial SIRT3: a new potential therapeutic target for metabolic syndrome. Mol Cell 2011; 44: 170-171.

40. Miao L, St Clair DK. Regulation of superoxide dismutase genes: implications in disease. Free Radic Biol Med 2009; 47: 344-356.

41. Qiu X, Brown K, Hirschey MD, Verdin E, Chen D. Calorie restriction reduces oxidative stress by SIRT3-mediated SOD2 activation. Cell Metab 2010; 12: 662-667.

42. Lopez-Lluch G, Hunt N, Jones B, Zhu M, Jamieson H, Hilmer S et al. Calorie restriction induces mitochondrial biogenesis and bioenergetic efficiency. Proc Natl Acad Sci USA 2006; 103: 1768-1773.

43. Fridell YW, Sanchez-Blanco A, Silvia BA, Helfand SL. Targeted expression of the human uncoupling protein 2 (hUCP2) to adult neurons extends life span in the fly. Cell Metab 2005; 1: $145-152$

44. Zhang $\mathrm{H}$, Zhang J, Ungvari Z, Zhang $\mathrm{C}$. Resveratrol improves endothelial function: role of TNF\{alpha\} and vascular oxidative stress. Arterioscler Thromb Vasc Biol 2009; 29: 1164-1171.

45. Wong RH, Howe PR, Buckley JD, Coates AM, Kunz I, Berry NM. Acute resveratrol supplementation improves flow-mediated dilatation in overweight/obese individuals with mildly elevated blood pressure. Nutr Metab Cardiovasc Dis 2011; 21: 851-856.

46. Park SJ, Ahmad F, Philp A, Baar K, Williams T, Luo H. Resveratrol ameliorates aging-related metabolic phenotypes by inhibiting cAMP phosphodiesterases. Cell 2012; 148: 421-433.

47. Aird WC. Phenotypic heterogeneity of the endothelium: I. Structure, function, and mechanisms. Circ Res 2007; 100: 158-173.

48. Aird WC. Phenotypic heterogeneity of the endothelium: II. Representative vascular beds. Circ Res 2007; 100: 174-190.

49. dela Paz NG, D'Amore PA. Arterial versus venous endothelial cells. Cell Tissue Res 2009; 335: 5-16.

50. Davidson SM, Duchen MR. Endothelial mitochondria: contribution to vascular function and disease. Circ Res 2007; 100: 1128-1141.

51. Waypa GB, Schumacker PT. Hypoxic pulmonary vasoconstriction: redox events in oxygen sensing. J Appl Physiol 2005; 98: 404-414.

52. Swift MR, Weinstein BM. Arterial-venous specification during development. Circ Res 2009; 104: $576-588$

53. Hewett PW. Vascular endothelial cells from human micro- and macrovessels: isolation, characterisation and culture. Methods Mol Biol 2009; 467: 95-111.

54. Moss SC, Bates M, Parrino PE, Woods TC. Isolation of endothelial cells and vascular smooth muscle cells from internal mammary artery tissue. Ochsner $J$ 2007; 7: 133-136.

55. Huang Q, Wu YT, Tan HL, Ong CN, Shen HM. A novel function of poly(ADP-ribose) polymerase- 1 in modulation of autophagy and necrosis under oxidative stress. Cell Death Differ 2009; 16: 264-277.

56. Ronnett GV, Ramamurthy S, Kleman AM, Landree LE, Aja S. AMPK in the brain: its roles in energy balance and neuroprotection. J Neurochem 2009; 109: 17-23.

57. Shin SM, Cho IJ, Kim SG. Resveratrol protects mitochondria against oxidative stress through AMP-activated protein kinase-mediated glycogen synthase kinase-3beta inhibition downstream of poly(ADP-ribose)polymerase-LKB1 pathway. Mol Pharmacol 2009; 76: 884-895.

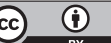

Cell Death and Disease is an open-access journal published by Nature Publishing Group. This work is licensed under a Creative Commons Attribution 4.0 International Licence. The images or other third party material in this article are included in the article's Creative Commons licence, unless indicated otherwise in the credit line; if the material is not included under the Creative Commons licence, users will need to obtain permission from the licence holder to reproduce the material. To view a copy of this licence, visit http://creativecommons.org/licenses/by/4.0 